\title{
IC 348 proper motion study from digitised Schmidt plates
}

\author{
R.-D. Scholz ${ }^{1}$, J. Brunzendorf ${ }^{2}$, G. Ivanov ${ }^{3}$, N. Kharchenko ${ }^{3}$, B. Lasker $^{4}$, H. Meusinger ${ }^{2}$, T. Preibisch $^{5,6}$, \\ E. Schilbach ${ }^{1}$, and H. Zinnecker ${ }^{1}$ \\ 1 Astrophysikalisches Institut Potsdam, An der Sternwarte 16, D-14482 Potsdam, Germany \\ e-mail: rdscholz@aip.de/eschilbach@aip.de/hzinnecker@aip.de \\ 2 Thüringer Landessternwarte Tautenburg, D-07778 Tautenburg, Germany \\ e-mail: brunz@tls-tautenburg.de/meus@obelix.tls-tautenburg.de \\ 3 Main Astronomical Observatory, Golosiiv, 252127 Kiev, Ukraine \\ e-mail: nkhar@mao.kiev.ua/ivanov@mao.kiev.ua \\ 4 Space Telescope Science Institute, 3700 San Martin Drive, Baltimore, MD 21218, U.S.A. \\ e-mail: lasker@stsci.edu \\ 5 Astronomisches Institut Universität Würzburg, Am Hubland, D-97074 Würzburg, Germany \\ e-mail: preib@astro.uni-wuerzburg.de \\ 6 Max-Planck-Institut für Radioastronomie, Auf dem Hügel 69, D-53121 Bonn, Germany
}

Received December 16, 1998; accepted March 25, 1999

\begin{abstract}
A new proper motion study for about 1400 stars with $R<18 \mathrm{mag}$ in a one square degree region around the young open cluster IC 348 based on Schmidt plates is presented. With an overall accuracy of about $3 \mathrm{mas} / \mathrm{yr}(2.5 \mathrm{mas} / \mathrm{yr}$, respectively for all stars with $R<$ $17 \mathrm{mag}$ ) we have obtained membership probabilities dividing our sample into three groups: foreground stars, cluster stars and background stars. This separation is also supported by the proper motion distribution with respect to the direction of the standard antapex and an increase of mean proper motion cluster membership probabilities with smaller cluster radii. 240 stars of our sample have cluster membership probabilities larger than $80 \%$. The results are compared with the highly accurate proper motion study of Fredrick (1956) and with other catalogues (PPM, ACT, Hipparcos), all containing only bright stars. From PPM and ACT proper motions a cluster radius of about 30 arcmin can be assumed. Hipparcos proper motions and parallaxes allow the separation of foreground stars, cluster stars and background stars in a somewhat larger region around IC 348 (with distances from the cluster centre of up to 85 arcmin). On the basis of Hipparcos data we calculate a mean distance of $261_{-23}^{+27}$ pc for the cluster stars with common proper motion. This is nearly the same distance as obtained by de Zeeuw et al. (1999) for the Per OB 2 association covering more than $15 \times 10$ square degrees. The mean proper motion of the cluster IC 348 obtained in our study is in good agreement with that of the highly probable members of the Per OB 2 association according to de Zeeuw et al. (1999). Therefore, we
\end{abstract}

Send offprint requests to: R.-D. Scholz conclude that the cluster IC 348 is embedded in the Per OB 2 association. From our proper motion membership probabilities we found a cluster radius of $10-$ 15 arcmin. There is a concentration of Fredrick's highly probable cluster stars just in between our cluster stars and the distant field stars proper motion distributions. On the other hand, two groups of stars in the proper motion diagram of Fredrick (1956) also seem to be indicated if all his possible cluster members are considered.

The X-ray sources from Preibisch et al. (1996) identified with optical counterparts from our proper motion sample show a strong concentration in the proper motion diagram. For these objects which are likely $\mathrm{T}$ Tauri stars we obtained high membership probabilities so that we conclude that they belong to the cluster IC 348 and to the Per OB 2 association. The proper motion foreground stars and cluster members which are counterparts of nearinfrared (NIR) sources (Lada \& Lada 1995) are located in different parts of the $(J-H)-(H-K)$ two colour diagram, respectively.

Key words: astrometry - methods: statistical open clusters and associations: individual: IC 348 stars: kinematics

\section{Introduction}

IC 348 is a very young cluster located near the edge of the Perseus molecular cloud complex at a distance of about 
300 pc (cf. Herbig 1998). Since the most massive star of the cluster (BD $31^{\circ} 643$ ) is only a B5V star, which is not powerful enough to disperse the cloud in which the stars were born, the cluster is still embedded in the remnant molecular core and most stars are obscured by 2 or more mag of visual extinction. The cluster was long believed to consist of just a dozen T Tauri members (Herbig 1954; Harris et al. 1954). However, recent sensitive observations have shown that it is much richer.

Preibisch et al. (1996) performed very deep ROSAT observations of IC 348 and could detect more than 100 $\mathrm{X}$-ray sources in a $1^{\circ}$ radius field. Many of these X-ray sources could be identified with $\mathrm{H} \alpha$ emitting T Tauri stars. The other X-ray sources very likely are T Tauri stars lacking strong $\mathrm{H} \alpha$ emission (weak-line $\mathrm{T}$ Tauri stars).

Herbig (1998) performed an optical study of IC 348. He could detect over $110 \mathrm{H} \alpha$ emission-line stars with $W(\mathrm{H} \alpha)$ $\gtrsim 3 \AA$ in the area, of which a majority are most likely T Tauri members of IC 348. Spectroscopic and photometric observations of about 80 stars in the central $8^{\prime} \times 10^{\prime}$ region enabled Herbig (1998) to place these stars into the HRD. A comparison with pre-main sequence evolutionary tracks showed that most of $\mathrm{H} \alpha$ emitting stars have ages in the range 0.7 to $3 \mathrm{Myr}$, with a mean of $1.3 \mathrm{Myr}$. This age is very well consistent with the kinematic age of the Per OB 2 association (1.3 Myr; cf. Blaauw 1952; de Zeeuw et al. 1999), to which IC 348 is thought to belong (Blaauw 1952).

IC 348 has also been extensively studied in the nearinfrared (NIR). Lada \& Lada (199) conducted a deep $J$, $H, K$ imaging survey of a $20^{\prime} \times 20^{\prime}$ field and could detect over 500 NIR sources. From comparison with several control fields they infer about 400 of these NIR sources to be probable cluster members. Luhman et al. (1998) obtained $K$-band spectra for nearly all stars in the $5^{\prime} \times 5^{\prime}$ core of IC 348.

Although the radius of the cluster core seems to be only about $4^{\prime}-5^{\prime}$, there is evidence for a more widely distributed population of T Tauri stars: Herbig (1998) found $\mathrm{H} \alpha$ emitting stars scattered all over the entire $\sim 15^{\prime} \times 25^{\prime}$ area he investigated. Because there is a steep rise in the number of emission-line stars between $W(\mathrm{H} \alpha)=10 \AA$ and the detection limit near $W(\mathrm{H} \alpha)=3 \AA$, there are probably many more $\mathrm{T}$ Tauri stars with weaker $\mathrm{H} \alpha$ emission in this area. These weak-line T Tauri stars outside the core of IC 348 are very hard to identify. In optical and infrared images they cannot be easily discerned from background field stars. The ROSAT data have an X-ray flux limit corresponding roughly to $V \lesssim 16$; this means that the $\mathrm{X}$-ray selected sample of $\mathrm{T}$ Tauri stars is complete only for stars of spectral type $\mathrm{K}$ and earlier, while most of the $\mathrm{M}$ type stars were not detected by ROSAT. It would be very desirable to include these still undetected weak-line $\mathrm{T}$ Tauri stars into the current sample of known $\mathrm{T}$ Tauri stars, since global properties of this interesting cluster like the mass function or the star formation history, could be determined more reliably.

A proper motion study might help to find the weak-line $\mathrm{T}$ Tauri stars in the outer regions of IC 348. It might also provide evidence for the presumption that IC 348 is part of the Per OB 2 association (cf. de Zeeuw et al. 1999). Unfortunately, not much was known about the kinematics of the stars in this region before our study. The only proper motion study of IC 348 performed so far is that of Frederick (1956), who classified 17 stars as probable cluster members. His membership investigation was based not only on highly accurate proper motions but also on the $V-(B-V)$ colour-magnitude diagram. His study was limited to 38 bright stars in a region of about one square degree around the cluster centre.

Here we present a new proper motion study from Schmidt plates and the determination of membership probabilities for a much larger number of stars with a limiting magnitude of about $R=18$. The observations and measurements are described in Sect. 2. With an accuracy comparable to that of the ACT proper motion catalogue, we are able to distinguish the cluster stars from near and distant field stars and to give an estimate of the cluster radius (Sect. 4). We compare the results with other proper motion catalogues which are up to now restricted to the bright stars $(V<13.5)$ in the field (Sect. 5). The connection of the cluster IC 348 with the Per OB 2 association is shown on the basis of Hipparcos distance estimates and of our proper motion and membership study (Sect. 5.4).

In addition we describe a $U B V R$ photographic photometry also based on the Schmidt plates (Sects. 3 and 7). The photographic photometry, however, suffers from large extinction variations (Snow et al. 1994) in the field, and was only thought to be a possible supplement to the main part of this work which is the proper motion study. A first comparison of our results with X-ray (Sect. 6) and NIR observations (Sect. 8) in the field around IC 348 is given.

A more detailed analysis of the cluster IC 348 based on our catalogue of proper motions, membership probabilities and photographic photometry combined with more accurate proper motions of the bright stars and accurate photometry from other catalogues will be subject of a next paper (Belikov et al., in preparation).

\section{Observations and measurements}

\subsection{Plate material}

For this study we used exclusively Schmidt plates from the Palomar Schmidt telescope (scale $67.2 \mathrm{arcsec} / \mathrm{mm}$ ), i.e. from the Palomar Observatory Sky Surveys (POSS) and from the Tautenburg Schmidt telescope (scale $51.4 \mathrm{arcsec} / \mathrm{mm})$. The plate material of Palomar $(\mathrm{P})$ and Tautenburg $(\mathrm{T})$ plates is described in Table 1.

For the proper motion study we selected a one square degree field centered on the cluster IC 348 
Table 1. Plate material

\begin{tabular}{|c|c|c|c|c|c|}
\hline $\begin{array}{l}\text { Telescope/ } \\
\text { Plate No. }\end{array}$ & $\begin{array}{l}\text { pass- } \\
\text { band }\end{array}$ & $\begin{array}{l}\text { epoch } \\
\text { [year] }\end{array}$ & $\begin{array}{l}\Delta t_{\exp } \\
{[\mathrm{min}]}\end{array}$ & $\begin{array}{l}\Delta x \\
{[\operatorname{arcm}}\end{array}$ & $\begin{array}{l}\Delta y \\
\text { in] }\end{array}$ \\
\hline $\mathrm{P} / \mathrm{o} 1457^{\mathrm{a}}$ & $O$ & 1955.81 & 12 & +100 & +100 \\
\hline $\mathrm{P} / \mathrm{e} 1457^{\mathrm{a}, \mathrm{b}}$ & $E$ & 1955.81 & 50 & +100 & +100 \\
\hline $\mathrm{P} / \mathrm{st} 277^{\mathrm{b}}$ & $V$ & 1982.80 & 20 & +99 & +100 \\
\hline $\mathrm{P} / \mathrm{sj} 01520^{\mathrm{b}}$ & $B_{j}$ & 1987.75 & 60 & -109 & +120 \\
\hline $\mathrm{P} / \mathrm{sf0} 4859^{\mathrm{b}}$ & $R$ & 1992.75 & 90 & -109 & +122 \\
\hline $\mathrm{T} / 9167$ & $U$ & 1997.09 & 90 & 0 & 0 \\
\hline $\mathrm{T} / 9168$ & $B$ & 1997.09 & 45 & 0 & 0 \\
\hline $\mathrm{T} / 9177$ & $B$ & 1997.10 & 40 & 0 & 0 \\
\hline
\end{tabular}

a Measurements taken from the APM sky catalogues.

b FITS frame from the STScI Digitized Sky Survey (DSS).

$\left(\alpha_{2000}=3^{\mathrm{h}} 44^{\mathrm{m}} 36.5, \delta_{2000}=32^{\circ} 8^{\prime} 59^{\prime \prime} .8\right)$. On all Palomar plates the cluster was located at about 1.5 to 2 degrees from the plate centre in each coordinate. The last two columns in Table 1 show the coordinate differences (cluster centre minus plate centre) on the plates. With a one square degree field around the cluster we do not use the plate edge zones which would be problematic for both astrometry and photometry. The Tautenburg plates were taken with the cluster in the plate centre. Due to rather poor weather conditions these plates do not go as deep as the Palomar plates.

Whereas for the older Palomar plates (POSS1 and Quick-Survey) as well as for the Tautenburg plates Kodak 103a-O or the similar ORWO ZU-21 emulsions were used, these are replaced by fine grain emulsions IIIa-J and IIIa-F in the POSS2 survey (Reid et al. 1991). According to Totten \& Irwin (1998) the POSS1 $E$ plate covers with $6200 \AA-6900 \AA$ a similar wavelength region as the POSS2 $R$ plate $(6300 \AA-6900 \AA)$. On the other hand, the POSS1 $O$ plate $(3400 \AA-4900 \AA)$ covers a passband comparable to $U+B$ and the POSS2 $B_{j}$ plate $(3900 \AA-5400 \AA)$ passband is also different from the standard $B$ passband. The Tautenburg plates were taken in the $U$ and $B$ passbands. Zero point shifts and colour terms in the transformation relations between $U$ and $B$ magnitudes of the Tautenburg system and the standard system are very small (van den Bergh 1964; Börngen \& Chatschikjan 1967). In the present study, the Tautenburg plates were therefore envisaged mainly for photometry. The transformation of the Palomar $B_{j}$ and $E$ magnitudes, respectively to $B$ and $R$ magnitudes will be subject of Sect. 3 .

\subsection{Measurements and determination of image parameters}

All Palomar Schmidt plates, except o1457 were measured with PDS microdensitometers at the Space Telescope Science Institute. The POSS1 plate e1457 and the
Quick-Survey plate st277 were measured with $25 \mu \mathrm{m}$ pixel size, whereas the POSS2 plates sj01520 and sf04859 were scanned with $15 \mu \mathrm{m}$ pixel size.

The POSS1 plates were also measured with the APM facility at Cambridge, UK (Kibblewhite 1984). In our study these APM measurements were mainly used for image identification and classification. We did not use them for the astrometry since these measurements were taken from the APM catalogues (Irwin et al. 1994) providing only one set of coordinates for both the $E$ and $O$ plates. This set of coordinates was produced using the $E$ plate as a reference plate so that for all objects measured on both plates only the coordinates as measured on the $E$ plate were preserved. Moreover, the blue POSS1 plate o1457 seemed to be very noisy with thousands of spurious images in the one square degree field detected by the APM.

The Tautenburg plates, which we used for photometry only, were scanned with the Tautenburg Plate Scanner (TPS) described by Brunzendorf \& Meusinger (1998). The TPS essentially consists of a rigid CCD line camera system with telecentric projection and an $x-y$ movable plate holder. Plates are digitised with $10 \mu \mathrm{m}$ resolution, corresponding to $0.5 \mathrm{arcsec} / \mathrm{pixel}$ on a Tautenburg plate.

For the object search and the determination of image parameters in the FITS frames we used the Münster Redshift Project (MRSP) software package (Horstmann et al. 1989). In its original version, this software executes a linear transformation between photographic densities and the logarithm of the corresponding intensities. For its application to Tautenburg plates a nonlinear transformation is used, based on either the characteristic curve of the individual plate or an average characteristic curve, for the transformation to intensities. The individual characteristic curve is derived from the calibration wedge exposed onto each plate. The use of such a nonlinear transformation ensures that the intensity profiles of stellar images are well-approximated by a Gaussian even when the central densities are outside the linear part of the characteristic curve. From all detected objects on each plate we selected for further reduction only those fitted by a twodimensional Gaussian profile. Measurements on a large number of plates of a different field have shown that the MRSP Gaussian fitting procedure (programme "profil", Horstmann et al. 1989) works well for stars over a magnitude interval of at least $13 \mathrm{mag}$ (Brunzendorf \& Meusinger 1999). In the present study we used only an 8 mag interval $(10.5<R<18.5)$.

The data from the APM catalogues include a classification of all measured objects into stars, objects with non-stellar image shape, merged objects and noise. The APM classification of stellar images is reliable until about two magnitudes above the plate limit. In order to exclude unreliably measured stars, we used this APM image classification on the reference plate (see Sect. 2.3) in addition 


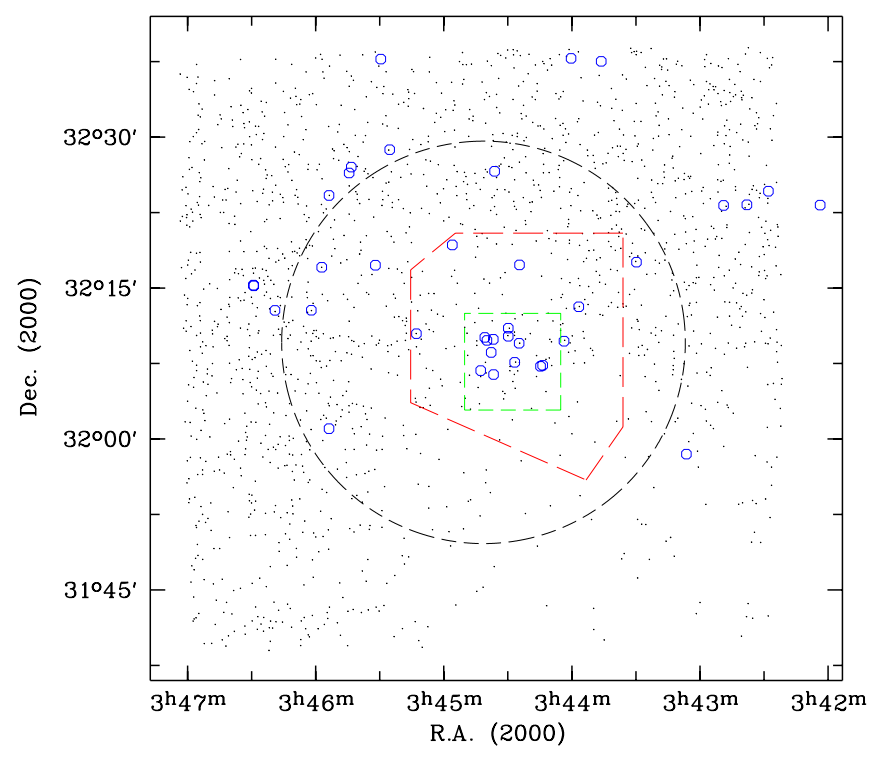

Fig. 1. The one square degree field around IC 348 selected for this study. Dots show all objects with Gaussian image profile on at least three plates for which proper motions have been determined. Small circles show the location of the 38 bright stars in the proper motion study of Fredrick (1956). The small dashed rectangle shows the region in which Trullols \& Jordi (1997) obtained $U B V R I$ photometry, the region shown by long dashed lines was covered by NIR photometry (Lada \& Lada 1995) and the dashed circle shows the main field of ROSAT observations (PSPC) used by Preibisch et al. (1996)

to the condition of a Gaussian profile as obtained by the MRSP software from the FITS file of the same plate.

Unreliable measurements were mainly due to strong image crowding of the stars with nebulosities and the internal reflection halo of $o$ Persei on the Schmidt plates. Therefore, many bright stars in the central region of IC 348 had to be excluded from further reduction.

\subsection{Plate matching}

For the determination of proper motions and of a $U B V R$ photographic photometry we compiled a catalogue of measurements from all comparison plates with respect to one reference plate. The plate matching is done iteratively starting with bright objects and large search radii (up to several $\mathrm{mm}$ ), finally iterating down to faint objects within a target search radius of about $75 \mu \mathrm{m}$, corresponding to 5 arcsec in the scale of the Palomar Schmidt plates. In the matching procedure only simple coordinate transformations were applied, i.e. an affine model $(2 \times 6$ plate constants) allowing for shift, rotation and scale factor.

The first epoch Palomar plate e1457 measured on both APM and PDS was chosen as the reference plate in the plate matching. This plate was selected because it was one of the deepest plates and the only first epoch plate which could be used in the proper motion determination. From about 2300 objects detected by the MRSP software in the one square degree FITS frame of the reference plate e1457, about 2000 stars could be fitted by a Gaussian. About 1800 of these stars were identified with Gaussian image shape objects on at least two other plates. The additional condition of stellar image classification on the reference plate as measured on the APM further restricted the number of objects in the final sample to 1431 stars.

The number of identifications of the stars of our final sample on the comparison plates depended on the limiting magnitude and the different passbands of the plates. Whereas nearly all of the 1431 stars in our sample were identified with stellar objects on the POSS2 $R$ and $B_{j}$ plates, about $77 \%$ were found on the POSS Quick-Survey $V$ plate. In contrast, only about $38 \%$ could be identified on the Tautenburg $B$ plates and even only $18 \%$ on the $U$ plate owing to the higher extinction at shorter wavelengths and the poor weather conditions during exposure.

\section{Photographic photometry}

With the available plate material we decided to carry out a photographic photometry in addition to the main aim of the project which was the proper motion study.

The instrumental magnitudes of all measured objects obtained by a two-dimensional Gaussian fitting procedure (MRSP programme "profil", Horstmann et al. 1989) were transformed to the standard system. This was done separately for each plate by using the CCD $U B V R I$ standards of Trullols \& Jordi (1997). The standards cover only the central region of the one square degree field around IC 348 (see Fig. 1).

The transformation of the instrumental system to the standard system was done by linear and quadratic polynomial fits using up to 60 standard stars. Unfortunately, we were not able to determine a proper colour equation for this transformation. An attempt to determine the colour term after first fitting the instrumental magnitudes on plate sj01520 with the $B$ standard magnitudes and then investigating the residual magnitude differences as a function of the $B-V$ colours given by Trullols \& Jordi (1997) did not yield satisfactory results. The colour equation

$B_{j}=B-0.28 \cdot(B-V) \quad-0.1 \leq(B-V) \leq 1.6$,

given by Blair \& Gilmore (1982) could not be used, since only 12 bright stars out of the 43 standard stars have colours in the range $(B-V) \leq 1.6$.

The photographic photometry on the Schmidt plates was a priori expected to be difficult since the central region of IC 348, where the standard stars are located (Fig. 1), is strongly affected by image crowding from bright stars and nebulosities. Moreover, as is obvious from the inspection of Fig. 1, there is strong extinction which is irregularly distributed over the field. Thus, the adopted transformation 
Table 2. Photometric calibration

\begin{tabular}{lcccc}
\hline $\begin{array}{l}\text { Telescope/ } \\
\text { Plate No. }\end{array}$ & $\begin{array}{c}\text { TJ97 } \\
\text { stars }\end{array}$ & $\begin{array}{c}\text { mag interval } \\
\text { of standards }\end{array}$ & model $^{\text {a }}$ & $\begin{array}{c}\text { accuracy } \\
\text { of fit }^{\mathrm{b}}\end{array}$ \\
\hline $\mathrm{P} / \mathrm{e} 1457$ & 60 & $11.0<R<18.0$ & $1^{\mathrm{c}}$ & 0.31 \\
$\mathrm{P} / \mathrm{st} 277$ & 24 & $11.9<V<19.6$ & 1 & 0.29 \\
$\mathrm{P} / \mathrm{sj} 01520$ & 43 & $12.4<B<21.5$ & 2 & 0.36 \\
$\mathrm{P} / \mathrm{sf} 04859$ & 60 & $11.0<R<18.0$ & 2 & 0.26 \\
$\mathrm{~T} / 9167$ & 14 & $12.1<U<19.2$ & 1 & 0.19 \\
$\mathrm{~T} / 9168$ & 30 & $11.6<B<19.6$ & 2 & 0.23 \\
$\mathrm{~T} / 9177$ & 29 & $11.5<B<19.7$ & 2 & 0.25 \\
\hline
\end{tabular}

a $1=$ linear, $2=$ quadratic polynomial fit.

b Dispersion of magnitude differences (standard - fitted).

c Fit consisted of two straight lines for $R<16$ and $R>16$.

between the instrumental system to the standard system is only of moderate accuracy. In Table 2 , the dispersions of the differences between standard magnitudes and fitted magnitudes of all stars used in the fitting procedure are listed. The Tautenburg plates provide a somewhat higher accuracy due to their better scale but have a brighter limiting magnitude (the blue Palomar plate goes about 2.0 magnitudes fainter compared to the two blue Tautenburg plates).

Comparing the $B$ magnitudes obtained from the Tautenburg plates 9168 and 9177 with the $B_{j}$ magnitudes obtained from the Palomar plate sj01520, we found large systematic differences (see Figs. 2a and 2b), especially for the bright stars $(B<17)$. At least a part of these differences is expected to arise from the moderate accuracy of the calibration procedure described above, especially from the omission of the colour term. As a test, we applied the colour equation from Eq. (1) but could not remove the systematic difference between the Palomar and Tautenburg $B$ magnitudes.

A further source of the differences may be the smaller number of bright $(B<16.5)$ standards used in the calibration of the blue Palomar plate (5 stars) compared to the blue Tautenburg plates (9 stars) and the relatively large error of the magnitude calibration for plate sj01520 (see Table 2). The systematic deviation of the Palomar $B_{j}$ from the Tautenburg $B$ magnitudes is very similar for both Tautenburg plates. Therefore, we have obtained a simple transformation of the Palomar $B_{j}$ magnitudes into the system of the mean $B$ magnitudes of the Tautenburg plates

$B_{\mathrm{jc}, \mathrm{sj} 01520}=0.9 \cdot B_{\mathrm{j}, \mathrm{sj} 01520}+1.8 \quad B_{\mathrm{j}, \mathrm{sj} 01520}<17$

from a linear polynomial fit using the stars with $B_{\mathrm{j}, \mathrm{sj} 01520}<17$ measured as well on both Tautenburg $B$ plates. For the faint stars $\left(B_{\mathrm{j}, \mathrm{sj} 01520} \geq 17\right)$ we have not applied any correction, since the Tautenburg $B$ magnitudes become unreliable at $B \sim 19$ (only one standard star with $B>19$ was used in the calibration), whereas there were still about 20 standard stars with
Table 3. Internal photometric accuracy for blue and red plates described by the standard deviation of magnitude differences after the correction of the $B_{j}$ and $E$ magnitudes (Eq. (2) and (3)) had been applied

\begin{tabular}{lrccc}
\hline $\begin{array}{l}\text { magnitude } \\
\text { difference }^{\mathrm{a}}\end{array}$ & $n^{\mathrm{b}}$ & mag interval & $\begin{array}{c}\text { mean } \\
\Delta \mathrm{mag}\end{array}$ & $\begin{array}{c}\text { st. } \\
\text { dev. }\end{array}$ \\
\hline$B_{9168}-B_{9177}$ & 446 & $12.3<B<19.4$ & +0.00 & 0.27 \\
$B_{9168}-B_{9177}$ & 259 & $12.3<B<18.0$ & +0.04 & 0.13 \\
$B_{\mathrm{jc}, \mathrm{sj} 01520}-B_{\mathrm{T}}$ & 444 & $12.3<B<19.4$ & +0.04 & 0.32 \\
$B_{\mathrm{jc}, \mathrm{sj} 01520}-B_{\mathrm{T}}$ & 257 & $12.3<B<18.0$ & -0.04 & 0.24 \\
$E_{\mathrm{c}, \mathrm{e} 1457}-R_{\mathrm{P} 2}$ & 1411 & $10.4<R<18.5$ & +0.02 & 0.24 \\
$E_{\mathrm{c}, \mathrm{e} 1457}-R_{\mathrm{P} 2}$ & 413 & $10.4<R<16.0$ & -0.03 & 0.21 \\
\hline
\end{tabular}

a $B_{\mathrm{T}}=\left(B_{9168}+B_{9177}\right) / 2, \quad R_{\mathrm{P} 2}=R_{\mathrm{sf0} 0859}$.

b Number of common stars in given magnitude interval.

$19<B<21.5$ used in the calibration of the blue Palomar plate.

Figures $2 \mathrm{a}$ and $2 \mathrm{~b}$ show the result before the correction of $B_{j}$ had been applied. In Fig. $2 \mathrm{~d}$ the $B$ magnitudes obtained from the two Tautenburg $B$ plates are compared. The internal accuracy of the blue magnitudes obtained from two Tautenburg $B$ plates and one Palomar $B_{j}$ plate can be described by the standard deviation of the magnitude differences (see Table 3). For the two Tautenburg $B$ plates we obtained a mean $B$ magnitude difference $\left(B_{9168}-B_{9177}\right)$ of +0.00 with a standard deviation of $0.27 \mathrm{mag}$ over the whole magnitude interval. After excluding the obviously bad measured/calibrated faint stars on the Tautenburg $B$ plates $(B>18$, as can be seen from Fig. 2d) we obtained a much better accuracy ( $0.13 \mathrm{mag}$ standard deviation). The general trend in Figs. 2a and $2 \mathrm{~b}$ can be roughly explained as follows: According to Eq. (1), the Tautenburg $B$ is expected to be a few tenth mag fainter than $B_{j}$ for typical $(B-V)$. The apparent turnoff of the $B$ (Tautenburg) $-B_{j}$ relation at fainter magnitudes is likely due to a selection effect near the Tautenburg plate limit. Stars scattered, at fixed $B_{j}$, below the mean relation, due to the colour equation and/or due to measuring errors, will be measured on both the Tautenburg plate and the Palomar plate. However, stars scattered above the mean relation will be missed, in general, on the Tautenburg plates.

The standard deviation of the differences between the corrected $B_{j}$ and the mean Tautenburg $B$ magnitudes is still the largest value among all possible plate-to-plate comparisons shown in Table 3. But it is better than one could expect from the accuracies of the external calibrations given in Table 2 .

Between the $E$ and $R$ magnitudes obtained, respectively from the red POSS1 and POSS2 plates there are also small systematic deviations. The $E$ magnitudes of the POSS1 plate e1457 can be transformed to the system of the POSS2 $R$ plate by Eq. (3).

$E_{\mathrm{c}, \mathrm{e} 1457}=-2.21+1.307 \cdot E_{\mathrm{e} 1457}-0.0103 \cdot E_{\mathrm{e} 1457}^{2}$. 

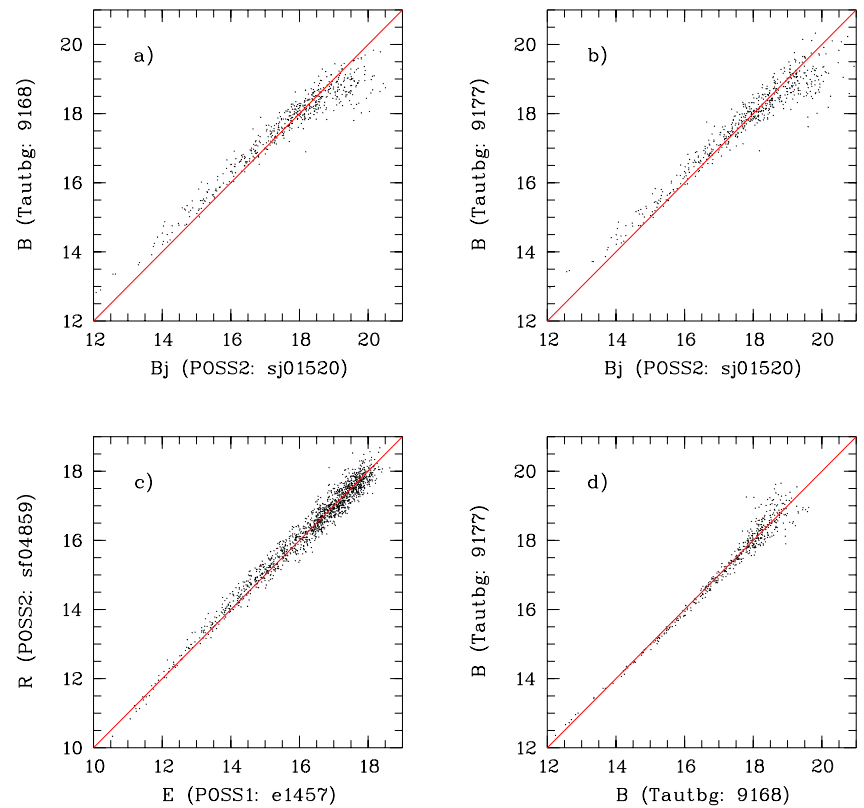

Fig. 2. Plate-to-plate comparison of calibrated photographic magnitudes (the solid lines indicate zero magnitude differences): a) and b) comparison of $B$ magnitudes obtained on the Tautenburg plates with $B_{j}$ magnitudes obtained on the POSS2 plate, c) comparison of POSS1 $E$ and POSS2 $R$ plates, d) Comparison of two Tautenburg $B$ plates. Figures a), b) and c) show the original calibrated magnitudes before the corrections (Eqs. (2) and (3)), respectively for the $B_{j}$ and $E$ magnitudes had been applied

Figure 2c shows the comparison between the red magnitudes before the transformation of the POSS1 $E$ magnitudes to the system of the POSS2 $R$ plate given by Eq. (3) had been applied.

After the correction of Eq. (3) we obtained, as an estimate of the internal magnitude accuracy, the mean difference $\left(E_{\mathrm{c}, \mathrm{e} 1457}-R_{\mathrm{sf04859}}\right)$ and its dispersion over the whole magnitude interval (see Table 3 ). The dispersion decreases not as strong as for the two Tautenburg $B$ plates if only the bright stars $(R<16)$ are considered.

\section{Determination of proper motions and cluster membership}

\subsection{Proper motion determination}

In the proper motion determination we followed the same principles as in the proper motion study in the field around the globular cluster M 3 (Scholz et al. 1997). The idea is to build up a time series of coordinates for each single object measured on $n$ plates. After the plate matching of all comparison plates with respect to one reference plate (the POSS1 plate e1457), all matched objects are used in plate-to-plate solutions for transforming the measured coordinates on the comparison plates into the coordinate system of the reference plate. The plate-to-plate solutions
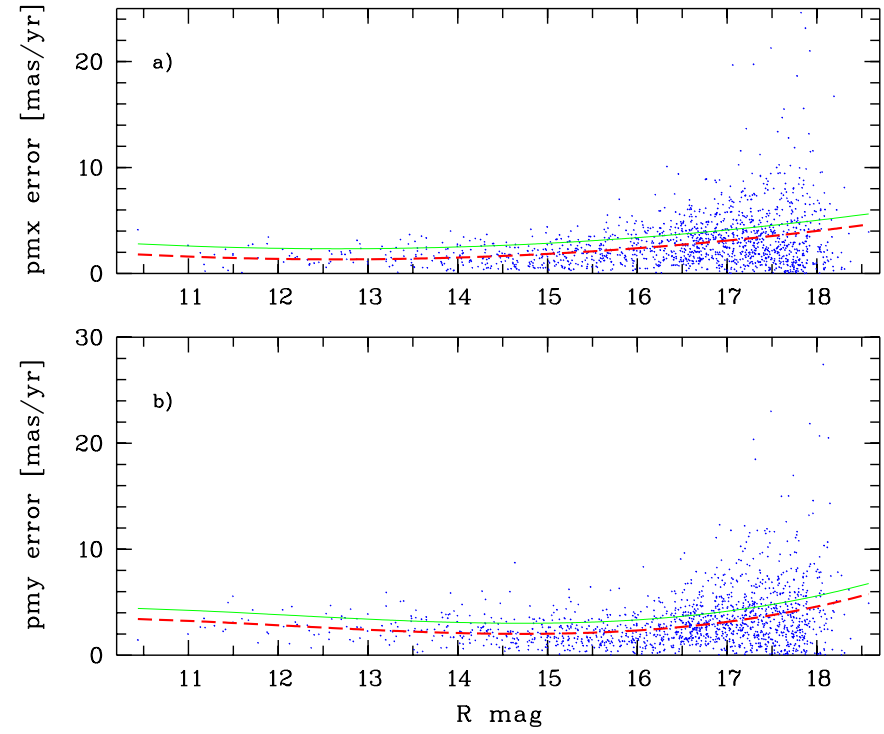

Fig. 3. Proper motion errors as a function of magnitude a) for $\sigma_{\mu_{x}}$ and $\left.\mathbf{b}\right)$ for $\sigma_{\mu_{y}}$. The dashed lines show the mean errors as obtained from a second order polynomial fit in case of $\sigma_{\mu_{x}}$ and from a third order polynomial fit in case of $\sigma_{\mu_{y}}$. The solid lines show the magnitude dependent proper motion dispersions $\sigma_{\mu_{x}}^{+1}(R)$ and $\sigma_{\mu_{y}}^{+1}(R)$ (obtained from adding $1 \mathrm{mas} / \mathrm{yr}$ to the mean errors) which were used in the determination of membership probabilities by Eq. (4)

are done with 3rd order polynomials and the method of stepwise regression described in Hirte et al. (1990). For the proper motion study we used only the Palomar plates measured with PDS measuring machines. Whereas for the blue POSS1 plate o1457 no independent astrometric measurements were available (see Sect. 2.2), we did not use the Tautenburg plates in the proper motion study since the epoch difference compared to the latest Palomar plate was only small and the Tautenburg plates did not go as deep as the Palomar ones.

Scholz et al. (1997) used exclusively Tautenburg Schmidt plates measured on the APM with a field of about $3^{\circ} \times 3^{\circ}$. For the absolute proper motion determination of the globular cluster Pal5, Scholz et al. (1998) combined Palomar, UKST and Tautenburg Schmidt plates measured on the APM using a smaller 1 square degree field. In both of these fields due to their high Galactic latitude large numbers of reference galaxies were available. Therefore, a zero point correction was applied by subtracting the mean coordinate difference of all galaxies measured on the comparison plate and on the reference plate before the proper motion determination.

In the low Galactic latitude field of IC $348\left(b \sim-18^{\circ}\right)$ with its large interstellar extinction no background galaxies are available so that we can only obtain relative proper motions. Therefore, we repeated the plate-to-plate solutions after a preliminary proper motion determination and selection of all objects with small proper motions 


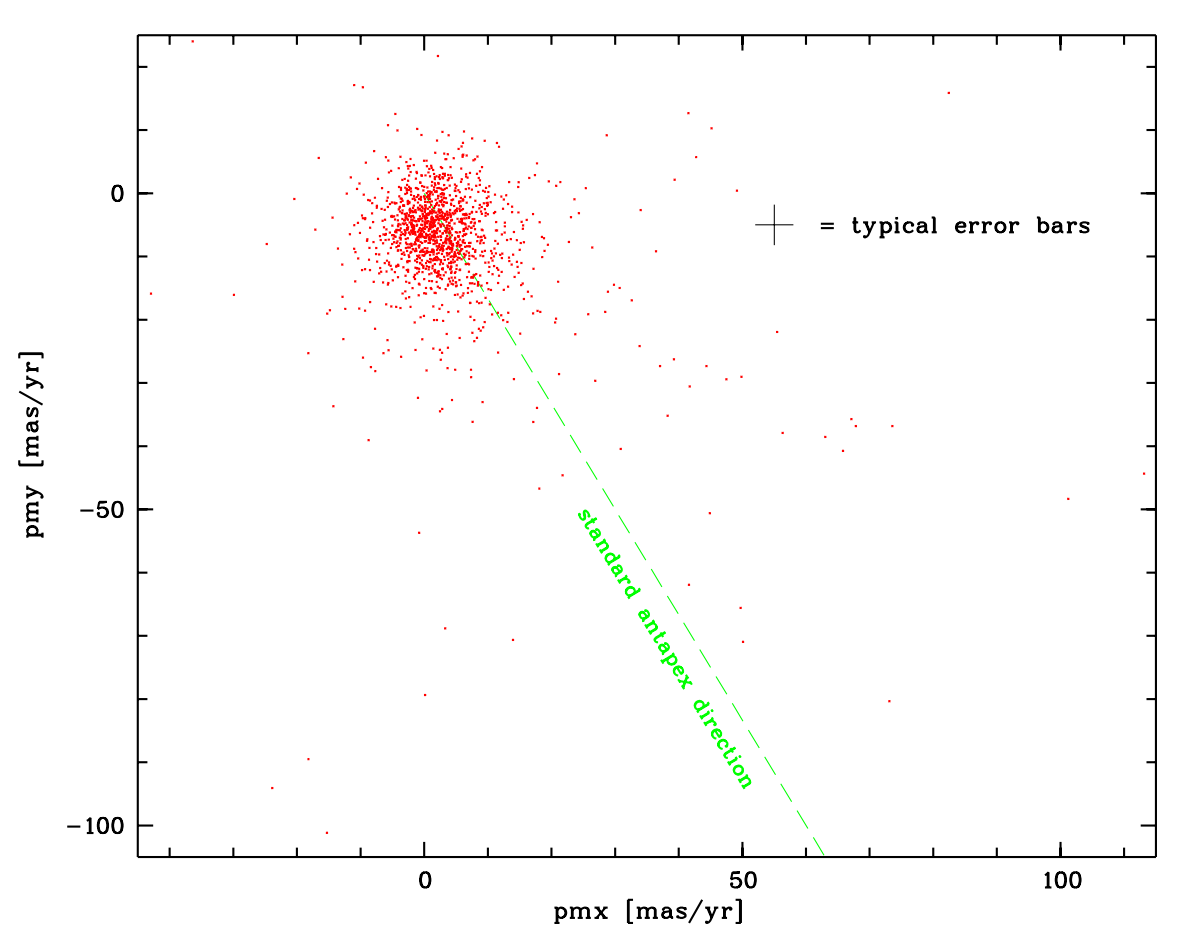

Fig. 4. Proper motions in the Hipparcos system of all 1431 stars of our sample in the one square degree field around IC 348. In the proper motion determination only the DSS frames of four POSS plates were used. $p m x$ corresponds to $\mu_{\alpha} \cos \delta, p m y$ corresponds to $\mu_{\delta}$. One can see a slightly elongated distribution in the direction of the standard antapex. The scale of the figure is the same as in Fig. 7 so that this proper motion diagram can be directly compared with those from other proper motion catalogues. The formal proper motion errors of our sample obtained from the linear regression of 4 (minimum 3) plate coordinates over the plate epochs are about $3 \mathrm{mas} / \mathrm{yr}$ in both components. This accuracy was confirmed by the comparison with the highly accurate proper motions from Fredrick (1956), see Sect. 5.5. The dependence of our proper motion errors on magnitude are shown in Fig. 3 $(|\mu|<15$ mas $/$ yr $)$. The relative proper motions of all objects with respect to the objects with small proper motions from the first run were then determined from the linear regression of the coordinates $(x, y)_{j}$ over the epochs $E p_{j}$, with $j=1 \ldots n_{\mathrm{pl}}$ where the number of plates $n_{\mathrm{pl}}$ for a given object is mainly dependent on its magnitude.

After the plate-to-plate solutions by stepwise regression with 3rd order polynomials and before the proper motion solution by linear regression of the coordinates over the epochs, we investigated the coordinate differences between each comparison plate and the reference plate for systematic effects as a function of the position of the stars in the field. No significant systematic errors were found. We repeated this error investigation taking the POSS2 $R$ plate as reference plate and did also not find significant systematic errors.

Different proper motion determinations by using different subsets of plates were carried out and the results compared with the proper motions of common bright stars from the catalogue of Fredrick (1956). Using 12 stars from Fredrick (1956) with $V>11$ (see Sect. 5.5) the dispersion of the proper motion differences decreased from about 4 mas/yr with the proper motion determination from one pair of plates (POSS1 + POSS2 $R$ plates) to about 3 mas/yr with three POSS plates (POSS1 + POSS2 $R$ plates + POSS Quick survey plate) and 2.5 mas $/ y r$ with all four available POSS plates.

Figure 4 shows the proper motion diagram of the complete sample of 1431 stars. Here and throughout the paper $\left(\mu_{x}, \mu_{y}\right)$ correspond to $\left(\mu_{\alpha} \cos \delta, \mu_{\delta}\right)$. The proper motions have been transformed to the Hipparcos system which is tied to the extragalactic reference system (Kovalevsky et al. 1997), in two steps (Sect. 5.5). First they were transformed to the system of Fredrick (1956) by Eq. (6) and then we applied the correction of the Fredrick system to the Hipparcos system given by Eq. (7).

The mean proper motion errors of all stars are $\sigma_{\mu_{x}}=$ $3.0 \mathrm{mas} / \mathrm{yr}$ and $\sigma_{\mu_{y}}=3.3 \mathrm{mas} / \mathrm{yr}$. Figure 3 shows how the proper motion errors change with the magnitudes of the stars. For all 820 stars with $R<17$ the mean proper motion errors are $\sigma_{\mu_{x}}=2.4 \mathrm{mas} / \mathrm{yr}$ and $\sigma_{\mu_{y}}=2.7 \mathrm{mas} / \mathrm{yr}$, respectively.

\subsection{Cluster membership}

Cluster membership can be estimated from kinematical data (i.e. proper motions and/or radial velocities), from the number density of the stars around an assumed cluster centre and from the location of the stars in observed colour-magnitude diagrams (CMDs). Distance measurements of sufficient numbers of member candidates of a cluster are usually not available to be included in membership studies. Hipparcos (ESA 1997) only recently provided an all-sky catalogue of proper motions and parallaxes for nearly 120000 stars with a limiting magnitude of $V \sim 12.4$, but completeness only to $V<8.5$. We will discuss in Sect. 5.4 how these data can be used for the case of IC 348 .

Fredrick (1956) discussed the membership of 38 bright stars around IC 348 for each star individually on the basis 
of its location in the proper motion diagram (see Fig. 7d), the position projected on the sky (see Fig. 1) and the location in the CMD (visual magnitudes versus $(B-V)$ colour indices, not shown here). Depending on the selection criteria, between 17 possible and 8 highly probable cluster members were found among the 38 stars.

Significantly larger numbers of member candidates were recently obtained on the basis of a near-infrared imaging survey (Lada \& Lada 1995), ROSAT X-ray observations (Preibisch et al. 1996) and $U B V R I$ CCD photometry (Trullols \& Jordi 1997). Lada \& Lada (1995) estimated that 380 NIR sources, i.e. the majority of the stars observed in their field of investigation (see Fig. 1), are members of the cluster. Preibisch et al. (1996) found 56 stars to be probable new members, presumably weak line $\mathrm{T}$ Tauri stars, because of their X-ray properties. Trullols \& Jordi (1997) classified 114 out of 123 stars investigated in the central region of IC 348 (see Fig. 1) as cluster members on the basis of colour-colour and colour-magnitude diagrams.

For the determination of membership probabilities of much more distant but well concentrated (in projected positions) globular clusters as well as open clusters on the basis of Schmidt plate measurements we have discussed and applied different methods (see Scholz \& Kharchenko 1994 for the globular cluster M 3, Kharchenko et al. 1997 for M 5 and Kharchenko \& Schilbach 1995 for a study of open clusters). In the last investigation of M 5 we determined membership probabilities of the globular cluster in a four-dimensional space of proper motions and coordinates using the maximum likelihood method. Different mean absolute proper motions and dispersions were found for three distribution functions of the cluster stars, near field stars and distant field stars. In addition to the fourdimensional distribution functions, we also investigated two-dimensional distribution functions including either only the proper motions or only the coordinates.

In the present case of IC 348 we decided not to include the coordinates and to investigate only two-dimensional distribution functions in the proper motions. The reason for that was the relatively bad measurement of the central cluster region on the Schmidt plates due to strong image crowding of bright stars and nebulosities as well as the apparently weak concentration of the fainter stars around the assumed cluster centre. In all other aspects we followed the same principles of the determination of the parameters of three distribution functions (foreground field stars, cluster stars, background field stars) by the maximum likelihood method as described in Kharchenko et al. (1997).

For the membership determination we selected from our catalogue of 1431 stars different subsamples of stars using the following criteria: We excluded stars with large proper motion errors $\sigma_{\mu}>\sigma_{\mu, \max }$, the faintest stars $R>$ $R_{\max }$, those with very large proper motions $\left(|\mu|>|\mu|_{\max }\right.$ in the original proper motion system, not yet transformed
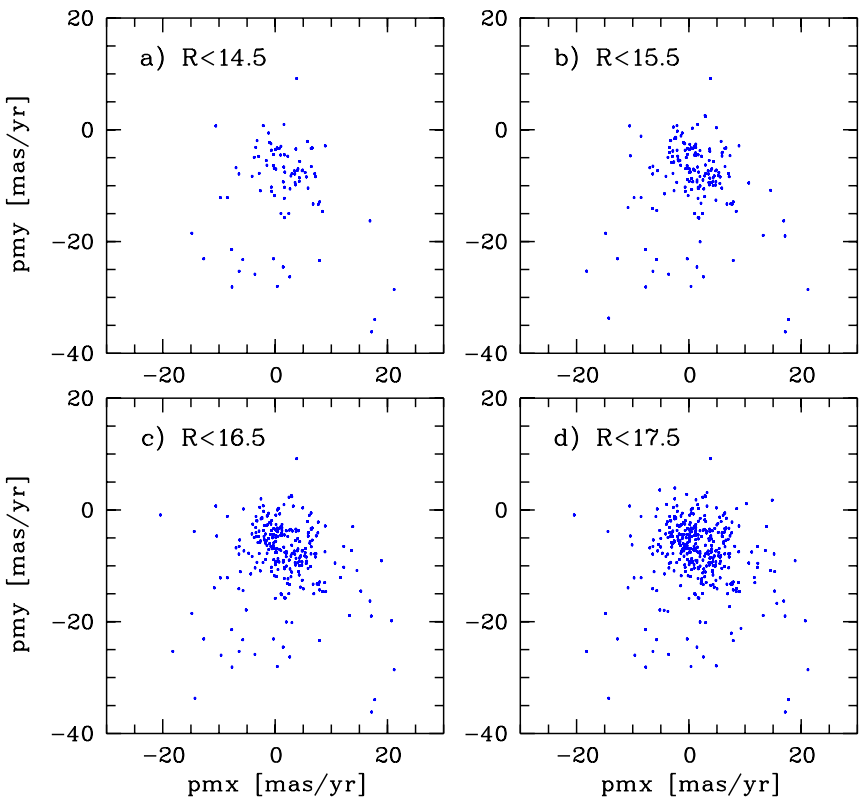

Fig. 5. Central part of proper motion diagram for different subsamples of stars used in the membership determination. Shown are the stars with proper motion errors $<4$ mas/yr, within a circular region around IC $348\left(r_{\max }=30 \mathrm{arcmin}\right)$ with different limiting magnitudes a) $R_{\max }=14.5$, b) $R_{\max }=15.5$, c) $R_{\max }=16.5$ and $\left.\mathbf{d}\right) R_{\max }=17.5$. The sample shown in $\mathbf{b}$ ) was finally selected for the determination of the parameters of three distribution functions (foreground field stars, cluster stars, background field stars) by the maximum likelihood method

by Eqs. (6) and (7) (see Sect. 5.5) and stars outside a preselected cluster radius $r>r_{\max }$.

The parameters of three distributions in the original proper motion system from the solution with 164 stars selected by $R_{\max }=15.5, \sigma_{\mu, \max }=4 \mathrm{mas} / \mathrm{yr},|\mu|_{\max }=$ $30 \mathrm{mas} / \mathrm{yr}$ and $r_{\max }=30 \mathrm{arcmin}$ are shown in Table 4. This solution was rather stable, i.e. not very sensitive to changes in the selection criteria. Particularly, the separation of two distributions with small dispersion of the same order but different mean proper motion and a third distribution with much larger dispersion was a characteristic feature of all solutions with different subsamples. Figure 5 shows as an example, how the proper motion diagram of accurately measured stars $\left(\sigma_{\mu, \max }=4 \mathrm{mas} / \mathrm{yr}\right)$ within a circular field around IC $348\left(r_{\max }=30 \mathrm{arcmin}\right)$ changes with the limiting magnitude $R_{\max }$.

Whereas the group $\mathrm{C}$ can easily be considered as near foreground stars, we have to decide which of the groups A and B represent the cluster IC 348 and which the more distant field stars. The dispersions of both groups A and B are comparable to the proper motion errors in our sample. In Table 4 we have also included the parameters of the three distributions after transforming all proper motions by Eqs. (6) and (7) to the Hipparcos system. 
Table 4. Mean proper motions (with their dispersion given in parentheses) of three distributions derived from a subsample of 164 preselected stars. A - cluster stars, B - distant field stars, C - foreground stars

\begin{tabular}{lccc}
\hline group & $n$ & $\begin{array}{c}\text { mean p.m. ( dispersion ) } \\
\mu_{x}\end{array}$ & $\mu_{y}$ \\
& & \multicolumn{2}{c}{ inas $/ y r]$} \\
\hline A & 82 & $+1.4(3.0)$ & $-2.8(3.2)$ \\
B & 52 & $-2.3(3.8)$ & $+1.1(3.8)$ \\
C & 30 & $-1.8(9.2)$ & $-13.5(6.2)$ \\
& \multicolumn{4}{c}{ in Hipparcos } \\
A & 82 & $+3.4(3.0)$ & $-9.3(3.2)$ \\
B & 52 & $-0.3(3.8)$ & $-5.4(3.8)$ \\
C & 30 & $+0.2(9.2)$ & $-20.0(6.2)$ \\
\hline
\end{tabular}

After this correction and taking into account the direction of the standard antapex, we interpret group A as a cluster at intermediate distance and group B as the more distant field stars. An additional strong argument supporting this interpretation is given by the different dependency of the two probabilities on the distance from the cluster centre (see Sect. 4.3, Fig. 6).

After the parameters of the distribution functions have been determined with a small subsample of stars, the probabilities for all stars to be a member of one of the obtained distributions were derived as:

$P=\exp \left(-0.25 \cdot\left(\left(\frac{\mu_{x}-\overline{\mu_{x}}}{\sigma_{\mu_{x}}^{+1}(R)}\right)^{2}+\left(\frac{\mu_{y}-\overline{\mu_{y}}}{\sigma_{\mu_{y}}^{+1}(R)}\right)^{2}\right)\right)$

where $\overline{\mu_{x}}$ and $\overline{\mu_{y}}$ are the mean proper motions of the distribution from Table 4 and $\mu_{x}, \mu_{y}$ are the individual proper motion of a star. Instead of the dispersions given in Table 4 we preferred to use the magnitude dependent dispersions $\sigma_{\mu_{x}}^{+1}(R)$ and $\sigma_{\mu_{y}}^{+1}(R)$ obtained from the mean proper motion errors plus 1 mas/yr (see Fig. 3). The magnitude dependent dispersions defined by this way vary between $\sim 3$ mas $/$ yr and $\sim 4$ mas/yr, respectively for $\sigma_{\mu_{x}}^{+1}(R)$ and $\sigma_{\mu_{y}}^{+1}(R)$ in the magnitude interval $10.5<R<16.5$ and rise up to $\sim 5$ mas $/$ yr and $\sim 6$ mas $/$ yr at $R=18$ (see Fig. 3).

Membership probabilities were determined for all 1431 stars of our proper motion sample, respectively determining the probability of each star to be a member of the cluster $P_{\mathrm{cl}}$ and of the distant field stars $P_{\mathrm{df}}$. For the foreground stars we have not determined a third membership probability criterion but defined them to have small values (e.g. $<5 \%$ ) for both $P_{\mathrm{cl}}$ and $P_{\mathrm{df}}$.

\subsection{Cluster radius}

With not including any information on the position of the stars in the membership determination (Sect. 4.2,
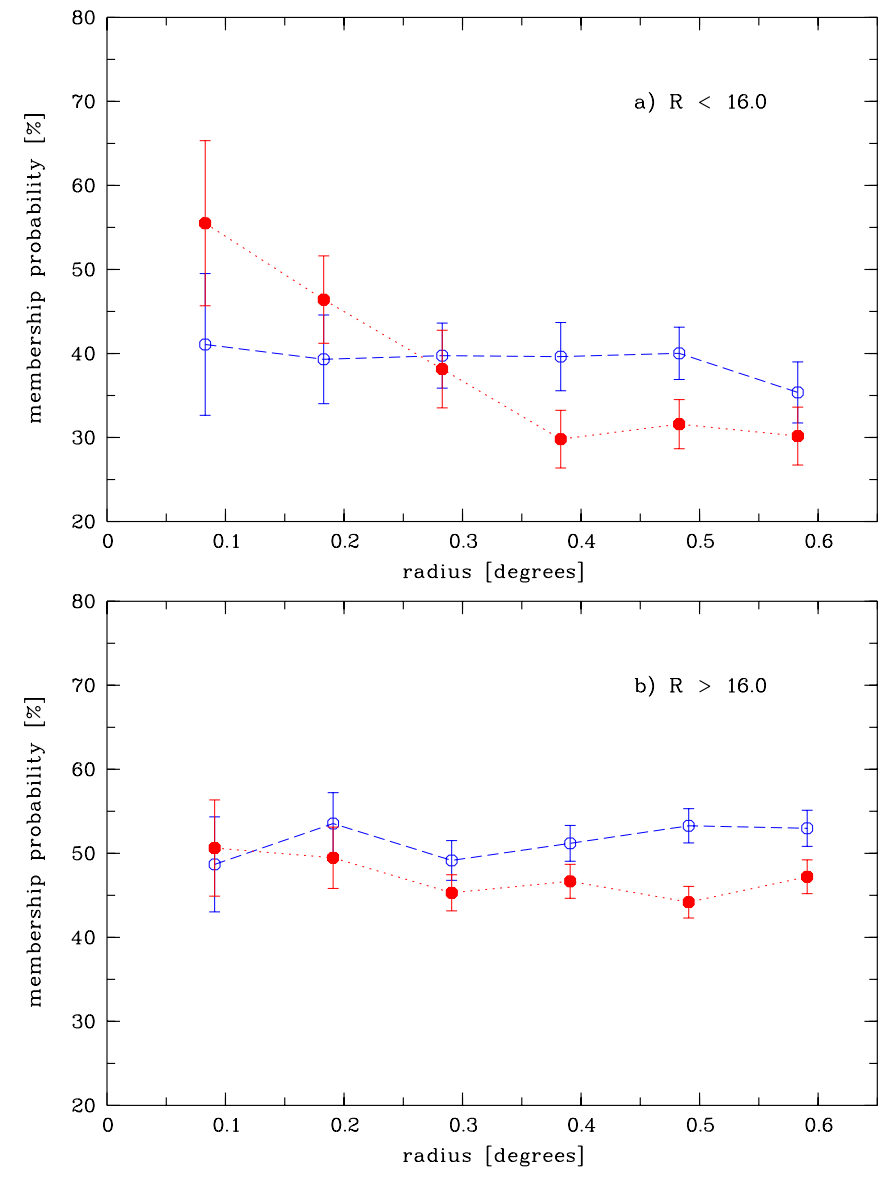

Fig. 6. Mean membership probabilities $P_{\mathrm{cl}}$ (filled circles, dotted lines) and $P_{\mathrm{df}}$ (open circles, dashed lines) of all stars in concentric rings around the assumed centre of IC 348 - a) for all stars with $R<16, \mathbf{b})$ for all stars with $R>16$

Eq. (4)), it is straightforward to investigate the proper motion membership probabilities as a function of the position in the field. To do so, we divided the field into concentric rings around the assumed cluster centre of IC 348 and computed the mean probabilities of all stars within a ring to be members of the cluster $\left(P_{\mathrm{cl}}\right)$ or of the distant field stars $\left(P_{\mathrm{df}}\right)$. If our interpretation of the two distributions A and B (Table 4), respectively as cluster stars and more distant field stars, is correct, we may expect no change of the mean probability $P_{\mathrm{df}}$ with the distance from the cluster centre. On the other hand we would expect a concentration of the cluster members in their positions, i.e. a decrease of the mean probability $P_{\mathrm{cl}}$ with the distance from the cluster centre.

Figure 6 shows the change of the mean probabilities $P_{\mathrm{cl}}$ (filled circles and dotted lines) and $P_{\mathrm{df}}$ (open circles and dashed lines) for the bright stars $(R<16)$ and faint stars $(R>16)$, respectively. As expected, the mean probability $P_{\mathrm{df}}$ does not show significant variation over the field. But for the mean cluster membership probabilities based only on the proper motion data and obtained without any 
positional parameters, we see a strong correlation with the distance from the cluster centre, if we look at the brighter stars (Fig. 6a). For the faint stars (Fig. 6b), there is only a small difference between the mean probabilities $P_{\mathrm{cl}}$ and $P_{\mathrm{df}}$ in dependence on the distance from IC 348. This is due to the larger overlap of the two membership probabilities obtained by Eq. (4) and the use of the magnitude dependent dispersions $\sigma_{\mu_{x}}^{+1}(R)$ and $\sigma_{\mu_{y}}^{+1}(R)$ instead of the constant dispersion given in Table 4.

In addition to the support of our proper motion cluster membership we obtained from the result in Fig. 6a, we can also estimate the cluster radius as $\sim 10-15$ arcmin. This is an important contribution to the discussion of the possible extension of the cluster IC 348 (cf. Herbig 1998; Lada \& Lada 1995).

The achieved accuracy of proper motions is not sufficient to substantiate a separation of the cluster into several subgroups as proposed by Lada \& Lada (1995) from their NIR photometry (see also Sect. 8).

\subsection{The catalogue}

The catalogue of positions $\alpha, \delta(2000)$, proper motions and their errors (in the Hipparcos system), the original $U, B, B_{j}, V, E$ and $R$ magnitudes as obtained from each individual plate (without corrections of Eqs. (2) and (3)), membership probabilities $P_{\mathrm{cl}}$ of the cluster IC 348 and $P_{\mathrm{df}}$ of the group of distant field stars, respectively are given for all 1431 stars. This table is only available in machine readable form from the CDS (cdsweb.u-strasbg.fr) via anonymous ftp.

\section{Comparison with other proper motion catalogues}

\section{1. $P P M$}

From the PPM catalogue (Röser \& Bastian 1991), the standard catalogue of positions and proper motions before Hipparcos, we found 49 stars in a circular region with a radius of 85 arcmin centered on IC 348 (see Fig. 8, PPM stars drawn as + ). For these 49 stars which are brighter than 12.5 (the PPM lists visual or photographic magnitudes) the average proper motion errors are $4.2 \mathrm{mas} / \mathrm{yr}$.

The proper motions of the PPM stars have been transformed to the Hipparcos system using the 20 Hipparcos stars in the field. All these Hipparcos stars were also found in the PPM catalogue. The accuracy of this transformation (Eq. (5) in mas/yr) was estimated as the mean error of the mean proper motion differences for these 20 stars.

$$
\begin{gathered}
\mu_{x}(\mathrm{PPM} \rightarrow \mathrm{Hipp})=\mu_{x}(\mathrm{PPM})+2.4 \pm 1.2 \\
\mu_{y}(\mathrm{PPM} \rightarrow \mathrm{Hipp})=\mu_{y}(\mathrm{PPM})+2.7 \pm 1.1
\end{gathered}
$$

The proper motion diagram of the PPM stars after the transformation to the Hipparcos system is shown in
Fig. 7a. There are 10 PPM stars within 30 arcmin from the cluster center drawn as filled circles. These stars show a remarkable concentration in the proper motion diagram compared to the remaining stars outside this region. If we exclude one outlier (number 22 in Fredrick 1956, which is a clear nonmember), the remaining group of 9 stars shows a proper motion dispersion of 4.0 mas/yr and $4.5 \mathrm{mas} / \mathrm{yr}$, respectively in $\mu_{x}$ and $\mu_{y}$, which is in good agreement with the catalogue errors. Therefore, from the PPM proper motions this group of 9 stars can be considered as a cluster with common proper motion (cf. Table 5).

\subsection{Starnet}

The Starnet catalogue (Röser 1996) gives proper motions determined from the combination of the improved Guide Star Catalogue (GSC 1.2, Röser et al. 1997) and the Astrographic Catalogue (AC). Unless the large epoch difference of about 80 years, the GSC 1.2 positions are only of moderate accuracy and, moreover, the accuracy of proper motions in the region of IC 348 suffers also from the low accuracy of AC positions in the declination zone north +30 deg. Nevertheless, we have selected Starnet data in the same large region around IC 348 (radius $85 \mathrm{arcmin}$ ) yielding an overall number of 214 stars with GSC magnitudes $<13.5$. For these stars the average proper motion errors were $6.6 \mathrm{mas} / \mathrm{yr}$, i.e. significantly larger than the errors of the present proper motion study and of other catalogue data used in this work. Therefore, we did not include these data in the discussion and a proper motion diagram is not shown here.

\subsection{ACT catalogue}

The Tycho catalogue (ESA 1997) provides positions of about 25 mas accuracy (at epoch 1991.25), for about one million stars with $V<11.5$ but only low accurate proper motions ( $\sim 25 \mathrm{mas} / \mathrm{yr})$. Combining the accurate positions (compared to other catalogues as PPM or GSC) with the measurements of the Astrographic Catalogue, made at the beginning of the century, one can get proper motions about ten times more accurate than the Tycho proper motions. This was done by Urban et al. (1998b), constructing the ACT catalogue after transforming the AC to the AC2000 on the Hipparcos system (Urban et al. 1998a).

From 69 Tycho stars within a 85 arcmin search radius around IC 348 ACT data are available for 65 stars. These stars plotted as $\times$ in Fig. 8 have average proper motion errors of $2.6 \mathrm{mas} / \mathrm{yr}$ and $3.0 \mathrm{mas} / \mathrm{yr}$, respectively for $\sigma_{\mu_{x}}$ and $\sigma_{\mu_{y}}$. The proper motions of these ACT stars (already in the Hipparcos system) are shown in Fig. $7 \mathrm{~b}$ with the stars of the central region around IC $348(r<30$ arcmin $)$ being plotted as filled circles. If we further exclude from 

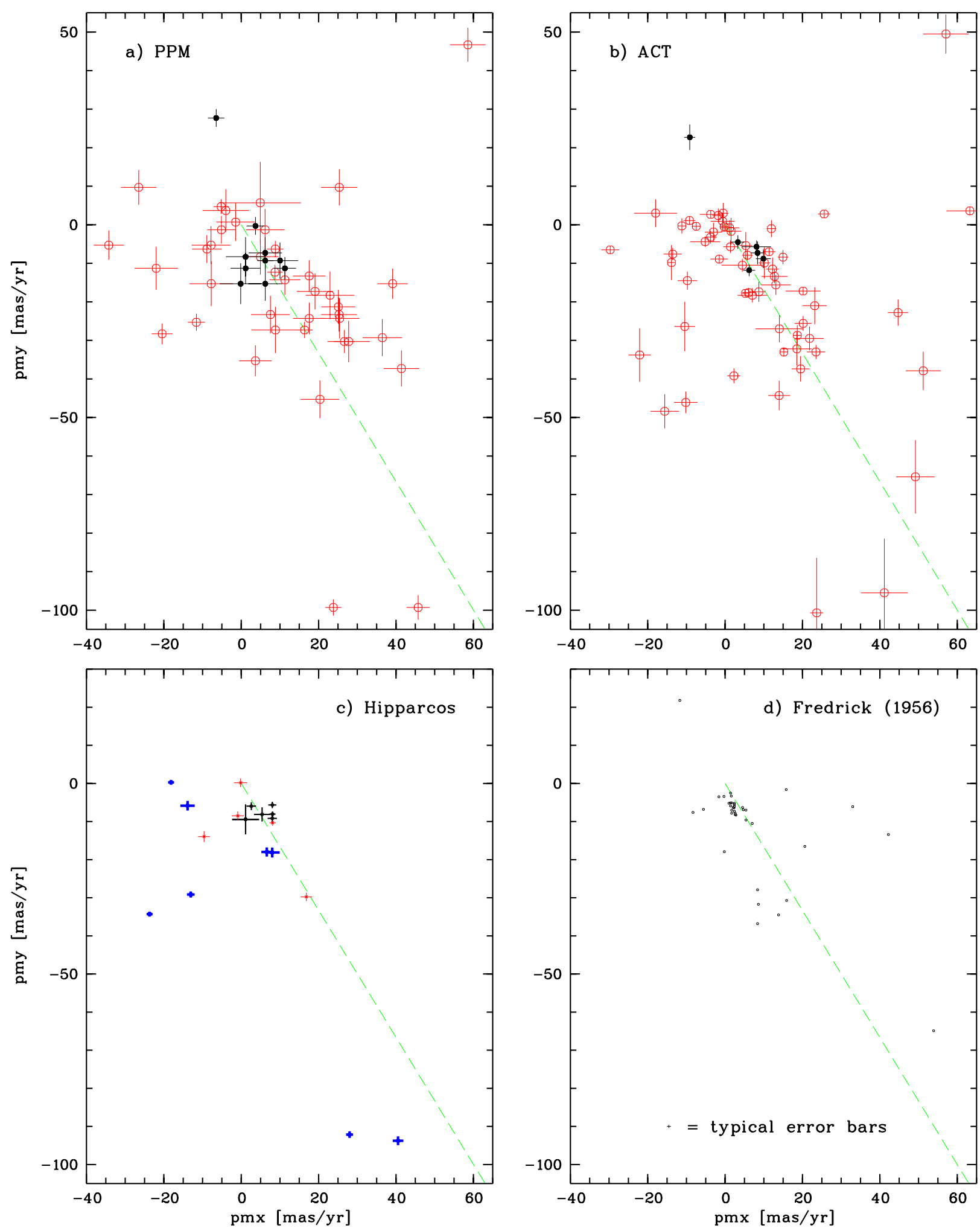

Fig. 7. Proper motions in Hipparcos system from different catalogues: PPM, ACT and Hipparcos stars within 85 arcmin from IC 348 are shown in a), b) and c). PPM and ACT stars with smaller distances from cluster centre $(r<30$ arcmin) are shown as filled circles in a) and b). Hipparcos stars with bold, normal and thin error bars, have parallaxes $\pi>5,3<\pi<5$ and $\pi<3$ mas, respectively. There is a remarkable separation of three groups of stars seen in the Hipparcos data: foreground stars with large proper motion dispersion, a group of stars at intermediate distances between $200 \mathrm{pc}$ and 320 pc with small proper motion dispersion (comparable to Hipparcos errors) and a third group of more distant stars which show again a larger proper motion dispersion. For comparison all stars from Fredrick (1956) are shown in d). Dashed lines show standard antapex direction 


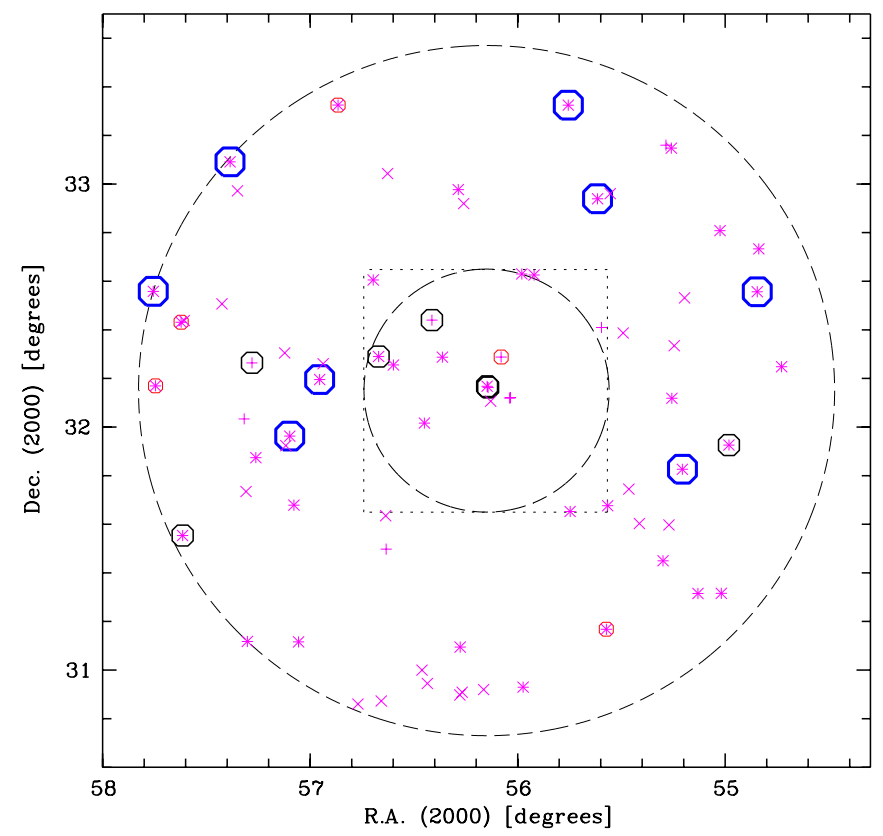

Fig. 8. Catalogue stars in an extended region around IC 348. The large dashed circle shows the search radius of 85 arcmin, the smaller one the cluster region with a radius of 30 arcmin. The dashed box indicates the one square degree field of the present study. 49 PPM stars found within 85 arcmin from IC 348 are drawn as,+ 64 ACT stars are drawn as $\times$. The 20 Hipparcos stars within 85 arcmin are drawn as circles of different size and line thickness in dependence on their measured parallaxes: large and bold circles for stars with $\pi>5$ mas, circles with medium size and line width for stars with $3<\pi<$ 5 mas, small and weak circles for stars with $\pi<3$ mas. One can see that the Hipparcos stars with intermediate distances, i.e. $3<\pi<5$ mas, which were also found as a common proper motion group (see Fig. 7d) are not concentrated in the cluster region with a radius of 30 arcmin but distributed over a larger field

the six stars in the central region one outlier in the proper motion diagram, the remaining five stars are well concentrated with a proper motion dispersion of 2.5 mas $/ \mathrm{yr}$ and $2.8 \mathrm{mas} / \mathrm{yr}$, respectively in $\sigma_{\mu_{x}}$ and $\sigma_{\mu_{y}}$. This proper motion dispersion is in very good agreement with the catalogue errors so that from the ACT data these five stars can be interpreted as members of a cluster. Table 5 shows the mean proper motion and dispersion of the 5 ACT stars in comparison to different member selections from other catalogues.

\subsection{Hipparcos}

Only five stars from the Hipparcos catalogue (ESA 1997) fall into the one square degree field of our investigation. Four of these stars were also included in the proper motion catalogue of Fredrick (1956). These stars are $o$ Persei, the brightest $(V=3.8, \mathrm{~B} 1 \mathrm{III})$ star in the one square
Table 5. Comparison of mean proper motions (with their dispersions given in parentheses) for different membership criteria applied to some catalogues

\begin{tabular}{lllll}
\hline Catalogue & $\begin{array}{l}\text { selection } \\
\text { criteria }\end{array}$ & $\begin{array}{c}n \text { mean p.m. (dispersion) } \\
\mu_{x} \\
{[\mathrm{mas} / \mathrm{yr}]}\end{array}$ \\
\hline PPM & $\begin{array}{l}r<30 \text { arcmin } \\
\text { without F22 }\end{array}$ & 9 & $+5.1(4.0)$ & $-9.7(4.5)$ \\
ACT & $\begin{array}{l}r<30 \text { arcmin } \\
\text { without F22 } \\
\text { Hipp. }\end{array}$ & 5 & $+7.2(2.5)$ & $-7.6(2.8)$ \\
& $\begin{array}{l}r<30 \text { arcmin } \\
(3<\pi<5) \text { mas }\end{array}$ & 4 & $+3.9(3.4)$ & $-8.8(0.8)$ \\
Hipp. & $\begin{array}{l}r<85 \text { arcmin } \\
(3<\pi<5) \text { mas }\end{array}$ & 7 & $+4.9(3.3)$ & $-8.0(1.6)$ \\
Hipp. & $\begin{array}{l}r<85 \text { arcmin } \\
\text { pm concentration } \\
\text { Fll possible }\end{array}$ & 9 & $+4.6(3.7)$ & $-8.3(1.6)$ \\
Fredrick & $\begin{array}{l}\text { members } \\
\text { highly probable }\end{array}$ & 8 & $+2.4(1.1)$ & $-6.5(1.6)$ \\
Fredrick & $\begin{array}{l}\text { members } \\
\text { member }\end{array}$ & & $-7.1(0.9)$ \\
\hline
\end{tabular}

degree field around IC 348, $\mathrm{BD}+31^{\circ} 643 \mathrm{a}$ and $\mathrm{BD}+31^{\circ} 643 \mathrm{~b}$ (Fredrick numbers 17 and 18) generally accepted as the brightest members of IC 348, and HD 281157 ( $V=10.2$, B5) which is number 24 of Fredrick and according to its proper motion also a member of IC 348. Unfortunately, the Hipparcos proper motions and parallaxes of $\mathrm{BD}+31^{\circ} 643 \mathrm{a}$ and $\mathrm{BD}+31^{\circ} 643 \mathrm{~b}$ are identical with large common errors of $\geq 3.5 \mathrm{mas} / \mathrm{yr}$ and $3.3 \mathrm{mas}$, respectively. These errors are much larger than those of the other stars in the field (compare proper motion error bars in Fig. 7c).

The average proper motion errors of all 20 Hipparcos stars found within 85 arcmin from IC 348 are 1.5 mas/yr and $1.3 \mathrm{mas} / \mathrm{yr}$, respectively in $\mu_{x}$ and $\mu_{y}$. The average parallax error of these 20 Hipparcos stars is 1.5 mas. If we select among these relatively bright stars in an extended region around IC 348 three groups with $\pi>5$ mas (8 stars), 3 mas $<\pi<5$ mas (7 stars) and $\pi<3$ mas (5 stars) and plot them in a proper motion diagram we can see a remarkable correlation between the proper motion dispersion and the distance (Fig. 7c). Whereas both foreground and background stars show larger proper motion dispersions, the group at intermediate distance shows a proper motion dispersion which is comparable to the Hipparcos proper motion errors. This is a strong indication that these 7 stars are members of a cluster.

Using all stars concentrated in the proper motion diagram shown in Fig. 7c at $\left(\mu_{x}, \mu_{y}\right)=(+5,-8)$ mas $/ y r$ within a circle of $6 \mathrm{mas} / \mathrm{yr}$ radius (i.e. 9 Hipparcos stars, see also Table 5) we calculate a mean distance of $261_{-23}^{+27}$ pc. This value for the cluster distance considering 9 Hipparcos stars with a mean proper motion of $\left(\mu_{x}, \mu_{y}\right)=(+4.6,-8.3) \mathrm{mas} / \mathrm{yr}$ within 85 arcmin from IC 348 is in good agreement with the recent discussion 
of the distance of the Per OB 2 association by de Zeeuw et al. (1999). They obtained on the basis of Hipparcos data mean distances of $318 \pm 27$ for the early-type members and $282 \pm 21$ for the late-type members of the association covering more than $15 \times 10$ square degrees on the sky.

We may conclude on the basis of these distance estimates that the cluster IC 348 is a part of the Per OB 2 association. From the comparison of the mean proper motion we get an additional argument for this assumption: The mean proper motion of 12 stars with $>95 \%$ membership probability in the Per OB 2 association (de Zeeuw et al. 1999) is $\left(\mu_{x}, \mu_{y}\right)=(+4.9,-7.5)$ mas/yr, respectively with a dispersion of $1.5,2.0)$ mas/yr. This result for the Per OB 2 association agrees well with our mean proper motion of the IC 348 cluster stars given in Table 4 .

In Table 5 mean proper motions and their dispersions for the Hipparcos cluster stars selected on the basis of parallax and location in the field around IC 348 or only of their proper motion are shown in comparison to other cluster member selections from different catalogues. From all results in Table 5 the Hipparcos results are in best agreement with our membership determination (group A in Table 4).

\subsection{The catalogue of Fredrick}

The proper motion catalogue of Fredrick (1956) contains 38 stars with visual magnitudes smaller than 13.5 covering a field around IC 348 which is nearly equal to the one square degree field selected for this study (see Fig. 1). His catalogue consists of two parts based on the observations made with the Sproul 24-inch refractor (34 plates with a scale of $18.87 \mathrm{arcsec} / \mathrm{mm}$ and a time baseline of 38 years) and with the McCormick 26-inch refractor (21 plates with a scale of $20.75 \mathrm{arcsec} / \mathrm{mm}$ and a time baseline of 32 years). The proper motion error of the averaged Sproul and McCormick data (21 stars) was given as $0.3 \mathrm{mas} / \mathrm{yr}$, whereas the proper motion errors of the stars based on either Sproul or McCormick data were estimated as $0.5 \mathrm{mas} / \mathrm{yr}$.

Using 12 common stars of the catalogue of Fredrick (1956) and our study we transformed our proper motion system to the Fredrick system by applying the corrections given in Eq. (6) (in mas/yr).

$\mu_{x}(\rightarrow$ Fredrick $)=\mu_{x}+0.8 \pm 0.8$

$\mu_{y}(\rightarrow$ Fredrick $)=\mu_{y}-1.6 \pm 0.7$.

In dependence on the different selection criteria (proper motion, position and location in the CMD) there are 17 possible members among the 38 stars of Fredrick (1956). These 17 possible members were also considered as cluster stars in Preibisch et al. (1996). Based on a more rigorous selection, particularly allowing only a small region for the cluster stars in their projected position, Fredrick (1956)

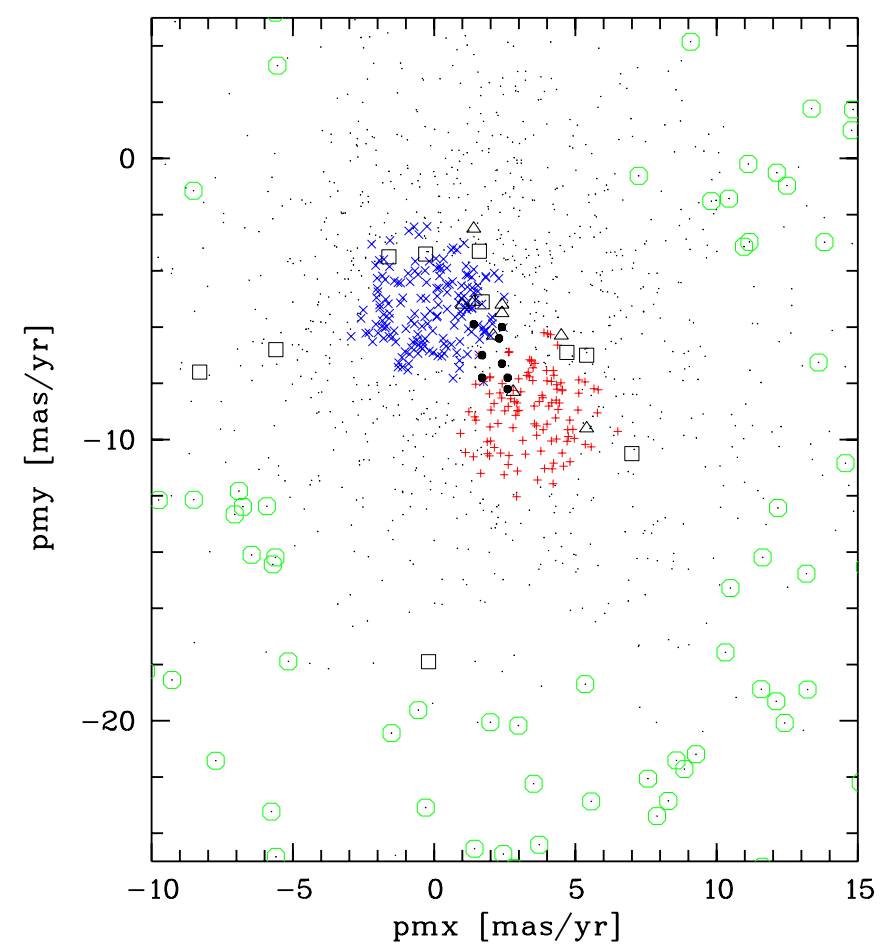

Fig. 9. Central part of proper motion diagram of our sample (in Hipparcos proper motion system) showing all measured proper motions as dots, the foreground stars with small proper motion membership probabilities of cluster and distant field stars $\left(P_{\mathrm{cl}}<10 \%\right.$ and $\left.P_{\mathrm{df}}<10 \%\right)$ overplotted as open circles, the distant field stars $\left(P_{\mathrm{df}}>90 \%\right)$ as small $\times$ and the cluster stars $\left(P_{\mathrm{cl}}>90 \%\right)$ as small + . In addition, the stars of Fredrick (1956) are shown including all highly probable (small filled circles) and other possible (open triangles) and some nonmembers (open squares) according to his proper motion study. As one can see the Fredrick members are located just in between the two distributions for cluster stars and distant field stars found in this study

discussed 8 highly probable members. Figure 9 shows the central region of the proper motion diagram with small dots representing our proper motion sample, overplotted open circles for the foreground stars (small proper motion membership probabilities for cluster and distant field stars, $P_{\mathrm{cl}}<10 \%$ and $P_{\mathrm{df}}<10 \%$ ), small $\times$ for the distant field stars $\left(P_{\mathrm{df}}>90 \%\right)$ and small + for the cluster stars $\left(P_{\mathrm{cl}}>90 \%\right)$. Also shown are the stars of Fredrick (1956) falling into this region of the proper motion diagram, including the 8 highly probable members (small filled circles), other 9 possible members (filled lozenges) and 10 nonmembers (filled squares).

The location of the cluster members according to Fredrick (1956) just in between the distributions of cluster stars and distant field stars determined in this study can not be explained by the accuracy of the transformation of our proper motions to the Fredrick system (Eq. (7)) which was sufficiently high. We are also quite sure about the 
significance of the two different distributions which we interpret as cluster stars and distant field stars (see Sects. 4.2 and 4.3). The elongated proper motion distribution even of the highly probable members of Fredrick (1956) may indicate somewhat larger errors in his $\mu_{y}$ proper motion components. On the other hand, this distribution of the possible proper motion members of Fredrick (1956) may also be an indication of two overlapping distributions instead of only one for the cluster stars. The elongation becomes even stronger and changes its direction from north-south to that of the standard apex direction if some additional stars near to the highly probable cluster stars in the proper motion diagram are included (compare Figs. 7d and 9).

There are 4 Hipparcos stars and 8 ACT stars among the 38 stars of the catalogue of Fredrick (1956) with one star having both Hipparcos and ACT proper motion measurements. We have used 4 Hipparcos plus 7 ACT proper motions to compute the corrections of the Fredrick proper motions to the Hipparcos system (given by Eq. (7) in $\operatorname{mas} / \mathrm{yr})$.

$\mu_{x}($ Fredrick $\rightarrow$ Hipp $)=\mu_{x}($ Fredrick $)+1.2 \pm 1.8$

$\mu_{y}($ Fredrick $\rightarrow$ Hipp $)=\mu_{y}($ Fredrick $)-4.9 \pm 0.7$.

Before that we have checked whether the ACT proper motions in the larger field around IC 348 (radius of $85 \mathrm{arcmin}$ ) show any residual deviation from the Hipparcos proper motion system and found no significant deviations (Eq. (8), in mas/yr) from 16 common stars in Hipparcos and ACT.

$$
\begin{aligned}
& \mu_{x}(\operatorname{Hipp})-\mu_{x}(\mathrm{ACT})=-0.3 \pm 0.7 \\
& \mu_{y}(\operatorname{Hipp})-\mu_{y}(\mathrm{ACT})=-0.1 \pm 0.8 .
\end{aligned}
$$

The relatively large error of the $\mu_{x}$ correction in Eq. (7) comes from the wide spread in the proper motion differences between Fredrick and ACT stars. Nevertheless, we did not use the alternative correction (Eq. (9) in mas/yr) obtained from only 4 Hipparcos stars.

$$
\begin{aligned}
& \mu_{x}(\text { Fredrick } \rightarrow \text { Hipp })=\mu_{x}(\text { Fredrick })+1.6 \pm 1.1 \\
& \mu_{y}(\text { Fredrick } \rightarrow \text { Hipp })=\mu_{y}(\text { Fredrick })-6.7 \pm 0.7 .
\end{aligned}
$$

In spite of the smaller formal errors of this correction, we decided that it would be less reliable due to the small number of 4 stars from which two (the double star $\left.\mathrm{BD}+31^{\circ} 643\right)$ have relatively large Hipparcos proper motion errors $(\geq 3.5 \mathrm{mas} / \mathrm{yr})$.

\section{Comparison with X-ray observations}

Preibisch et al. (1996) detected 116 X-ray sources in deep pointings with the ROSAT Position Sensitive Proportional Counter (PSPC) and the ROSAT High Resolution Imager

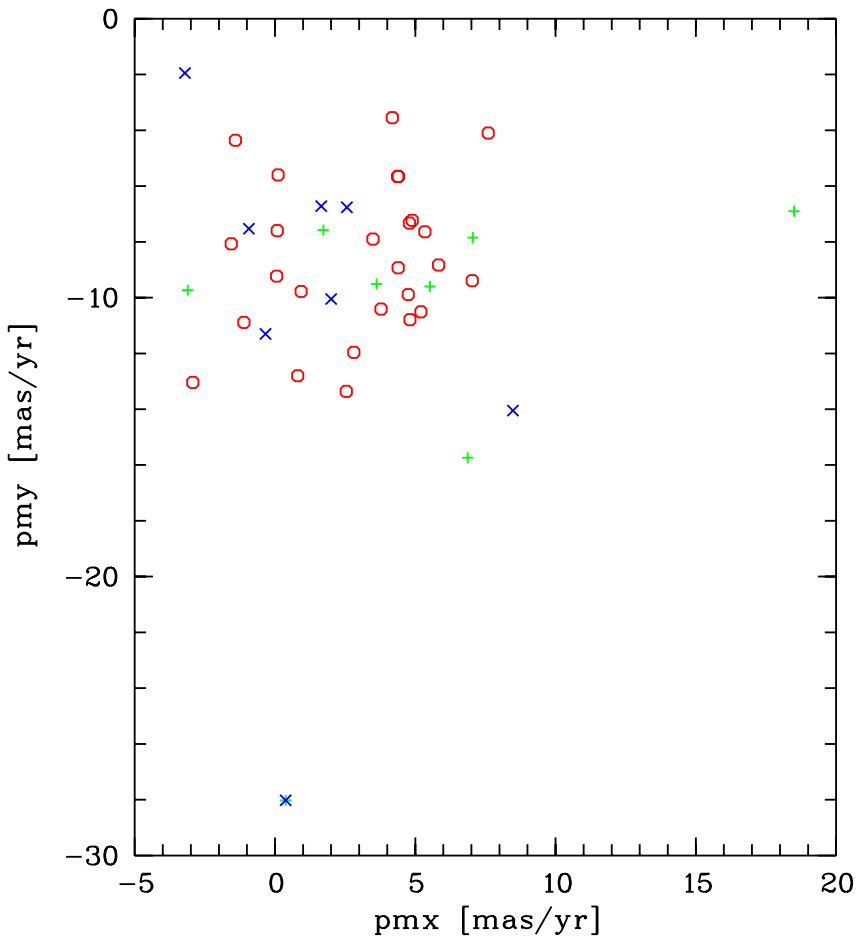

Fig. 10. Proper motions of starlike objects (from our sample of 1431 objects) identified with X-ray sources from Preibisch et al. (1996). Small circles show the 26 objects found within 5 arcsec search radius, additional identifications by increasing the search radius to $10 \operatorname{arcsec}(8$ objects) and $15 \operatorname{arcsec}(8$ objects), respectively are shown by + and $\times$. Two close X-ray sources were identified with the same object in the proper motion sample

(HRI). From these 116 sources we identified 26 with optical counterparts from our sample of 1431 objects using a search radius of 5 arcsec. By increasing the search radius to 10 arcsec and 15 arcsec, respectively we found additional $8+8$ identifications, with one identification of the same star in the proper motion sample with two different X-ray sources. The relatively small number of optical counterparts compared to the identification of Preibisch et al. (1996) is due to the selection criteria for our sample of starlike objects (Sect. 2.3).

The 26 X-ray sources identified within 5 arcsec with optical sources show a strong concentration in the proper motion diagram (see Fig. 10) at $\mu_{x}=2.9(2.9) \mathrm{mas} / \mathrm{yr}$ and $\mu_{y}=-8.6(2.7)$ mas $/ y r$ (mean values with their dispersions given in parentheses). These mean values and dispersions are in good agreement with those obtained for the cluster stars in our membership determination (see group $\mathrm{A}$ in Table 4) and with the mean proper motion and dispersion of the Per OB 2 association (Sect. 5.4).

Most of the X-ray sources identified with optical sources are within the $20^{\prime} \times 20^{\prime}$ field (see Fig. 11). All $\mathrm{X}$-ray sources within this small field were classified as cluster members by Preibisch et al. (1996). 


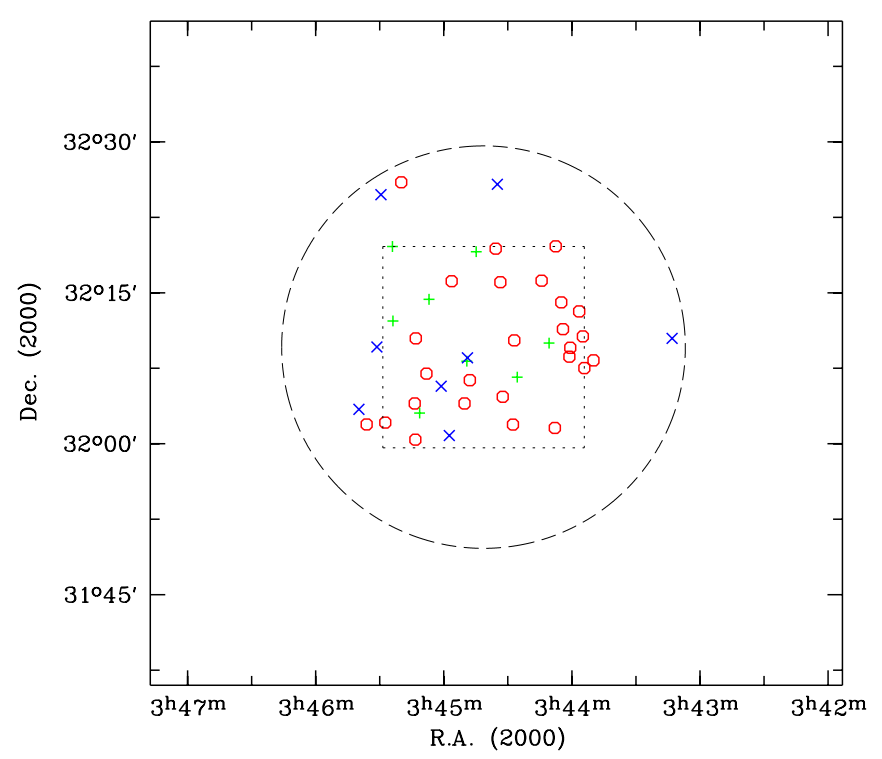

Fig. 11. The one square degree field around IC 348 with all X-ray sources from Preibisch et al. (1996) identified with optical sources from our sample of 1431 stars. The dashed line shows the main field of the ROSAT PSPC observations, open circles show the 26 objects found within 5 arcsec search radius, additional identifications by increasing the search radius to $10 \operatorname{arcsec}(8$ objects) and $15 \operatorname{arcsec}(8$ objects), respectively are shown by + and $\times$. All X-ray sources within the small $20^{\prime} \times 20^{\prime}$ field (dotted square) were classified as members of IC 348 by Preibisch et al. (1996)

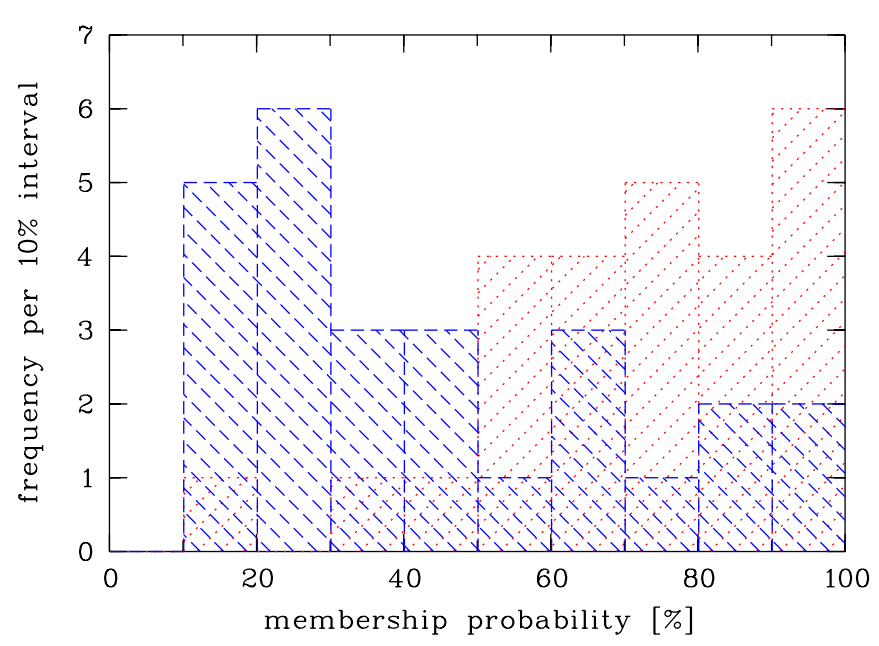

Fig. 12. Histogram of membership probabilities $P_{\mathrm{cl}}$ (dotted line) and $P_{\mathrm{df}}$ (dashed line) for the $26 \mathrm{X}$-ray sources identified within 5 arcsec with optical sources from our proper motion survey
Note that the scale in Fig. 10 is the same as in Fig. 9 but the start and end values of the two plots are different. Figure 9 does not show the proper motion dispersion of cluster and distant field stars but the highly probable members of both distributions $\left(P_{\mathrm{cl}}>90 \%\right.$ and $\left.P_{\mathrm{df}}>90 \%\right)$.

The distribution of the membership probabilities $P_{\mathrm{cl}}$ (dotted line) and $P_{\mathrm{df}}$ (dashed line) for the $26 \mathrm{X}$-ray sources identified within 5 arcsec is shown in Fig. 12. There are no foreground stars $\left(P_{\mathrm{cl}}<5 \%\right.$ and $\left.P_{\mathrm{df}}<5 \%\right)$ among the 26 $\mathrm{X}$-ray sources. Their cluster membership is more probable than a membership in the group of distant field stars.

\section{Comparison with colour-magnitude diagrams}

As expected, there is a large scatter in the photographic $(B-V)$ colours compared to those of the CCD photometry of Trullols \& Jordi (1997). Therefore, the photographic $V-(B-V)$ CMD can hardly be used as an additional tool in the membership determination and is not shown here. Obviously, the fluctuation of extinction in $B$ (and $V$ ) over the one square degree field and the variation of the sky background connected with photographic effects make the photographic $V-(B-V)$ CMD nearly useless. Note that the standard stars used for our photographic photometry are concentrated in the very central part of the field (see Fig. 1).

The situation becomes slightly better with the photographic $V-(V-R)$ CMD shown in Fig. 13 in comparison with the CCD photometry of Trullols \& Jordi (1997). Here the scatter in the photographic $(V-R)$ colours is not as large in comparison to the standard stars CCD photometry, unless small systematic deviations comparable to the errors of the photographic photometry can be seen. A more detailed analysis of these effects will be subject of a forthcoming paper (Belikov et al., in preparation).

\section{Comparison with NIR observations}

From 510 objects from the deep near-infrared survey of Lada \& Lada (1995) in their survey area indicated in Fig. 1 we were able to identify 44 optical counterparts in our sample of 1431 stars using a search radius of 2 arcsec. For 41 of these stars the proper motion errors are $<10 \mathrm{mas} / \mathrm{yr}$, for 28 stars they are $<5$ mas/yr. If we define the cluster members with proper motion membership probabilities $>50 \%$ and the foreground stars, respectively with small membership probabilities of both cluster stars and distant field stars $\left(P_{\mathrm{cl}}<5 \%\right.$ and $\left.P_{\mathrm{df}}<5 \%\right)$ we get 30 IC 348 members and 5 foreground stars among the optical counterparts of NIR sources.

Figure 14 shows the location of these optical counterparts in the two-colour diagram of Lada \& Lada (1995). One can see that the proper motion members of IC 348 


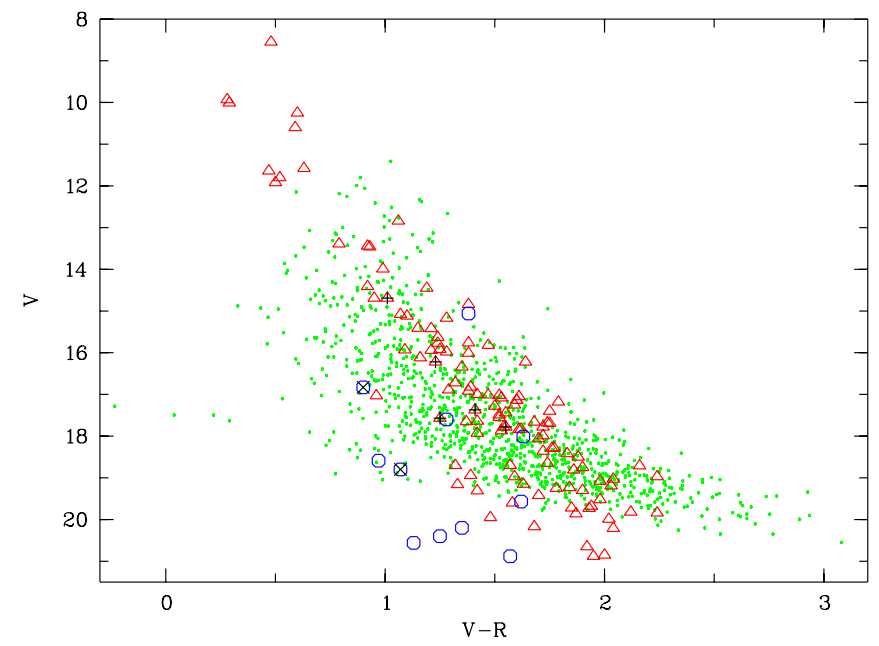

Fig. 13. Colour-Magnitude diagram $V-(V-R)$ for all 1083 objects (dots) from our sample of 1431 stars with magnitudes and colours obtained from the POSS Quick-Survey $V$ plate and the average of two red POSS plates after the POSS1 $E$ plate magnitudes were transformed to the POSS2 $R$ plate system by Eq. (3). For comparison, the stars from Trullols \& Jordi (1997) are plotted with their CCD magnitudes as open triangles (members according to their classification) and open circles (nonmembers). Five out of 25 stars from Trullols \& Jordi (1997) which are in the proper motion sample are clear nonmembers (drawn as + ) according to our proper motions $\left(P_{\mathrm{cl}}<5 \%\right)$ but were classified as members by Trullols \& Jordi (1997). On the other hand two of these 25 standard stars (drawn as $\times$ ) classified as nonmembers by Trullols \& Jordi (1997) may be members according to our proper motion data $\left(P_{\mathrm{cl}}>50 \%\right)$

tend to be redder in both colours $(H-K)$ and $(J-H)$, but especially in $(J-H)$ in comparison to the foreground stars. The members show also a larger dispersion in their colours than the foreground stars. This is in agreement with the comparison of the NIR photometry of Lada \& Lada (1995) in the field of IC 348 with two control fields outside the one square degree region around IC 348.

If we allow for a larger uncertainty in the positions of the NIR sources, e.g. using search radii of 3 and 4 arcmin, we identify 70 and 97 sources, respectively.

\section{Summary}

We have successfully used digitised sky survey data of the Palomar Schmidt telescope for a new proper motion study for about 1400 stars with $R<18 \mathrm{mag}$ in a one square degree region around the young open cluster IC 348. An overall accuracy of about 3 mas/yr for the stellar proper motions allowed to separate the cluster stars from both foreground and background stars. Whereas the foreground stars could obviously be separated due to their large proper motion dispersion, the separation of the cluster

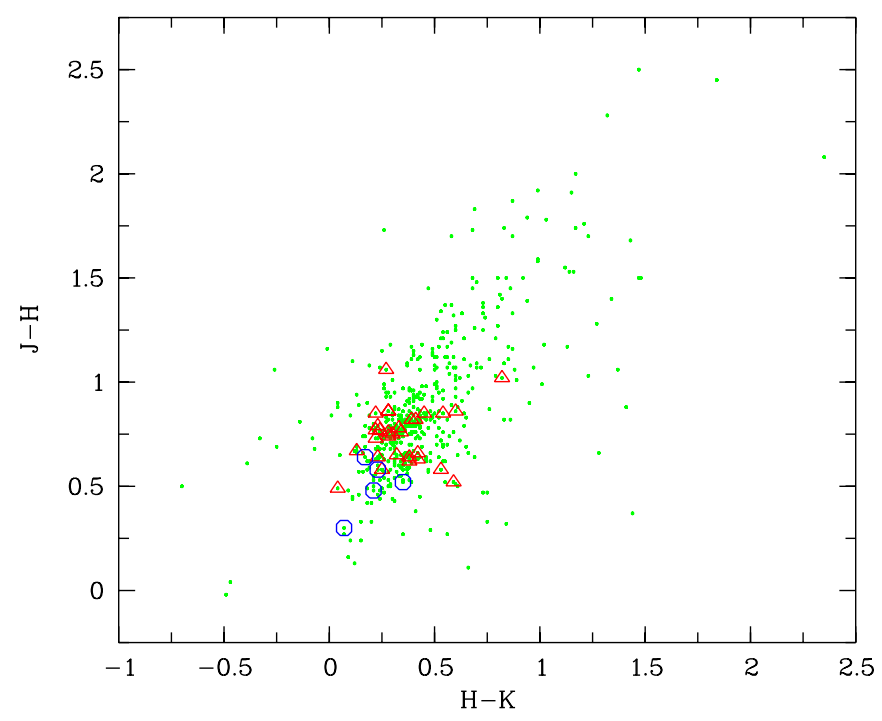

Fig. 14. Near-infrared two colour diagram for 510 objects (dots) from Lada \& Lada (1995). From 44 objects with optical counterparts in our sample of 1431 stars (search radius 2 arcsec) those with proper motion cluster membership probabilities $P_{\mathrm{cl}}>50 \%$ (30 objects) are overplotted as open triangles whereas the 5 proper motion foreground stars (cluster membership probabilities $P_{\mathrm{cl}}<5 \%$ and distant field stars membership probabilities $\left.P_{\mathrm{df}}<5 \%\right)$ are overplotted as open circles

from background stars was supported by the direction of the standard antapex and an increase of mean proper motion cluster membership probabilities with smaller cluster radii. A cluster membership probability of more than $80 \%$ was found for 240 stars of our sample.

We have compared our results with the highly accurate proper motion study of Fredrick (1956) and with other catalogues (PPM, ACT, Hipparcos), all containing only bright stars. On the basis of Hipparcos data we calculate a mean distance of $261_{-23}^{+27} \mathrm{pc}$ for the cluster. Hipparcos proper motions and parallaxes do also allow the separation of foreground stars, cluster stars and background stars in a somewhat larger region around IC 348. The mean proper motion of the cluster IC 348 obtained in our study is in good agreement with that of the highly probable members of the Per OB 2 association according to de Zeeuw et al. (1999). Together with the above mentioned Hipparcos distance estimate, which is also in agreement with de Zeeuw et al. (1999) data for Per OB 2, this confirms that IC 348 is embedded in the Per OB 2 association.

From our proper motion membership probabilities we found a cluster radius of $10-15$ arcmin. There is a concentration of Fredrick's highly probable cluster stars just in between our cluster stars and the distant field stars proper motion distributions. On the other hand, two groups of stars in the proper motion diagram of Fredrick (1956) also seem to be indicated if all his possible cluster members are considered. 
In addition to the proper motion study we have also carried out $U B V R$ photographic photometry using standard stars from Trullols \& Jordi (1997). Since the standards cover only the very central region of the one square degree field, the variation in extinction over the field lead to a large scatter in the photographic colours, particularly in $(B-V)$.

The X-ray sources from Preibisch et al. (1996) identified with optical counterparts from our proper motion sample show a strong concentration in the proper motion diagram. For these objects which are likely T Tauri stars we obtained high membership probabilities so that we conclude that they belong to the cluster IC 348 and to the Per OB 2 association. The proper motion foreground stars and cluster members which are counterparts of nearinfrared (NIR) sources (Lada \& Lada 1995) are located in different parts of the $(J-H)-(H-K)$ two colour diagram, respectively.

Acknowledgements. This research is based on data from the Digitized Sky Surveys and from the Tautenburg Schmidt telescope. The Digitized Sky Surveys were produced at the Space Telescope Science Institute under U.S. Government grant NAG $\mathrm{W}-2166$. The images of these surveys are based on photographic data obtained using the Oschin Schmidt Telescope on Palomar Mountain. We would like to thank the Palomar and Tautenburg observatories for supplying the plates used in this investigation.

This study has also made use of the APM catalogues (Irwin et al. 1994). The APM is a national astronomy facility financed by the Particle Physics and Astronomy Research Council.

We would also like to thank H.-J. Tucholke and R. Ungruhe for their support with the Münster (MRSP) software.

We are grateful to E. Lada, who provided us with the data of her NIR survey of IC 348, and to S. Röser for providing us with his Starnet catalogue data.

GI and NK thank the Deutsche Forschungsgemeinschaft (DFG) for financial support. JB gratefully acknowledges financial support from the DFG under grant Me 1350/3-2.

We thank the anonymous referee for useful comments and suggestions.

\section{References}

Blaauw A., 1952, Bull. Astron. Inst. Netherlands 11, 405 Blair M., Gilmore G., 1982, PASP 94, 742

Börngen F., Chatschikjan E., 1967, Astron. Nachr. 289, 19

Brunzendorf J., Meusinger H., 1998, in: The Message of the Angles - Astrometry from 1798 to 1998, Proc. Internat. Spring Meeting Astronomische Gesellschaft, Gotha 1998, Brosche P., Dick W.R., Schwarz O., Wielen R. (eds.).
Verlag Harri Deutsch, Thun and Frankfurt am Main, p. 148 Brunzendorf J., Meusinger H., 1999, in: Treasure Hunting in Astronomical Plate Archives, la Dous C. et al. (eds.). Verlag Harri Deutsch, Thun and Frankfurt am Main (in press)

de Zeeuw P.T., Hoogerwerf R., de Bruijne J.H.J., Brown A.G.A., Blaauw A., 1999, AJ 117, 354

ESA, 1997, Hipparcos and Tycho catalogues, ESA SP-1200

Fredrick L.W., 1956, AJ 61, 437

Harris D.L., Morgan W.W., Roman N.G., 1954, ApJ 119, 622

Herbig G.H., 1954, PASP 66, 19

Herbig G.H., 1998, ApJ 497, 736

Hirte S., Dick W.-R., Schilbach E., Scholz R.-D., 1990, in: Errors, Uncertainties and Bias in Astronomy, Jaschek C., Murtagh F. (eds.). Cambridge University Press, p. 343

Horstmann H., Schücker P., Seitter W., et al., 1989, Bull. Inf. CDS 37, 43

Irwin M.J., Maddox S.J., McMahon R., 1994, Spektrum No. 2, p. 14

Kharchenko N., Schilbach E., 1995, Astron. Nachr. 316, 91

Kharchenko N., Scholz R.-D., Lehmann I., 1997, A\&ASS 121, 439

Kibblewhite E., Bridgeland M., Bunclark P., Cawson M., Irwin M., 1984, in: Proc. of Conference on Astronomy with Schmidt-Type Telescopes, Capaccioli M. (eds.). Reidel Publ., p. 89

Kovalevsky J., Lindegren L., Perryman M.A.C., et al., 1997, A\&A 323, 620

Lada E.A., Lada C.J., 1995, AJ 109, 1682

Luhman K.L., Rieke G.H., Lada C.J., Lada E.A., 1998, ApJ 508,347

Preibisch T., Zinnecker H., Herbig G.H., 1996, A\&A 310, 456

Reid I.N., Brewer C., Brucato R.J., et al., 1991, PASP 103, 661

Röser S., Bastian U., 1991, PPM Star Catalogue 1, 2. Spektrum Akademischer Verlag, Heidelberg, Berlin, New York

Röser S., 1996, in: Proc. IAU Symp. 172, Ferraz-Mello S., Morando B., Arlot J.-E. (eds.). Kluwer, Dordrecht, p. 481

Röser S., Morrison J., Bucciarelli B., Lasker B., McLean B., 1997, in: Proc. IAU Symp. 179, McLean B.J., Golombek D.A., Hayes J.E., Payne H.E. (eds.). Kluwer, Dordrecht, p. 420

Scholz R.-D., Kharchenko N., 1994, Astron. Nachr. 315, 73

Scholz R.-D., Meusinger H., Irwin M., 1997, A\&A 325, 457

Scholz R.-D., Irwin M., Odenkirchen M., Meusinger H., 1998, A\&A 333, 531

Snow T.P., Hanson M.M., Seab C.G., Saken J.M., 1994, ApJ 420,632

Totten E.J., Irwin M.J., 1998, MNRAS 294, 1

Trullols E., Jordi C., 1997, A\&A 324, 549

Urban S.E., Corbin T.E., Wycoff G.L., Martin J.C., Jackson E.S., Zacharias M.I., Hall D.M., 1998a, AJ 115, 1212

Urban S.E., Corbin T.E., Wycoff G.L., 1998b, AJ 115, 2161

van den Bergh S., 1964, AJ 69, 610 